\title{
Second-Order Asymptotics for the Gaussian MAC with Degraded Message Sets
}

\author{
Jonathan Scarlett, Member, IEEE and Vincent Y. F. Tan, Senior Member, IEEE
}

\begin{abstract}
This paper studies the second-order asymptotics of the Gaussian multiple-access channel with degraded message sets. For a fixed average error probability $\varepsilon \in(0,1)$ and an arbitrary point on the boundary of the capacity region, we characterize the speed of convergence of rate pairs that converge to that boundary point for codes that have asymptotic error probability no larger than $\varepsilon$. As a stepping stone to this local notion of second-order asymptotics, we study a global notion, and establish relationships between the two. We provide a numerical example to illustrate how the angle of approach to a boundary point affects the second-order coding rate. This is the first conclusive characterization of the second-order asymptotics of a network information theory problem in which the capacity region is not a polygon.
\end{abstract}

\section{Index Terms}

Gaussian multiple-access channel, Degraded message sets, Superposition coding, Strong converse, Finite blocklengths, Second-order coding rates, Dispersion.

\section{INTRODUCTION}

In this paper, we revisit the Gaussian multiple-access channel (MAC) with degraded message sets. This is a communication model in which two independent messages are to be sent from two sources to a common destination; see Fig. 1 One encoder, the cognitive or informed encoder, has access to both messages, while the uninformed encoder only has access to its own message. Both transmitted signals are power limited, and their sum is corrupted by additive white Gaussian noise (AWGN).

The capacity region $\mathcal{C}$, i.e. the set of all pairs of achievable rates, is well-known (e.g. see [1, Ex. 5.18(b)]), and is given by the set of rate pairs $\left(R_{1}, R_{2}\right)$ satisfying

$$
\begin{aligned}
R_{1} & \leq \mathrm{C}\left(\left(1-\rho^{2}\right) S_{1}\right) \\
R_{1}+R_{2} & \leq \mathrm{C}\left(S_{1}+S_{2}+2 \rho \sqrt{S_{1} S_{2}}\right)
\end{aligned}
$$

for some $\rho \in[0,1]$, where $S_{1}$ and $S_{2}$ are the admissible transmit powers, and $\mathrm{C}(x):=\frac{1}{2} \log (1+x)$ is the Gaussian capacity function. The capacity region $\mathcal{C}$ does not depend on whether the average or maximal error probability formalism is employed, and no time-sharing is required. The region $\mathcal{C}$ for $S_{1}=S_{2}=1$ is illustrated in Fig. 2, observe that $\mathcal{C}$ is formed from a union of trapezoids, each parametrized by $\rho$. The vertical line segment corresponds to $\rho=0$, while the curved part corresponds to $\rho \in(0,1]$. The direct part of the coding theorem for $\mathcal{C}$ is proved using superposition coding [2].

While the capacity region is well-known, there is substantial motivation to understand the second-order asymptotics for this problem. For any given point $\left(R_{1}^{*}, R_{2}^{*}\right)$ on the boundary of the capacity region, we study the rate of convergence to that point for an $\varepsilon$-reliable code. More precisely, we characterize the set of all $\left(L_{1}, L_{2}\right)$ pairs, known as second-order coding rates [3]-[6], for which there exist sequences of codes whose asymptotic error probability does not exceed $\varepsilon$, and whose code sizes $M_{1, n}$ and $M_{2, n}$ behave as

$$
\log M_{j, n} \geq n R_{j}^{*}+\sqrt{n} L_{j}+o(\sqrt{n}), \quad j=1,2 .
$$

J. Scarlett was with the Department of Engineering, University of Cambridge, Cambridge CB2 1PZ, U.K. He is now with the Laboratory for Information and Inference Systems, École Polytechnique Fédérale de Lausanne, CH-1015, Switzerland (email: jmscarlett@gmail.com).

V. Y. F. Tan was with the Institute for Infocomm Research $\left(I^{2} \mathrm{R}\right)$, Agency for Science, Technology and Research (A*STAR). He is now with the Department of Electrical and Computer Engineering and the Department of Mathematics, National University of Singapore. (email:vtan@nus.edu.sg).

This paper was presented in part at the 2014 IEEE International Symposium on Information Theory in Honolulu, HI. 


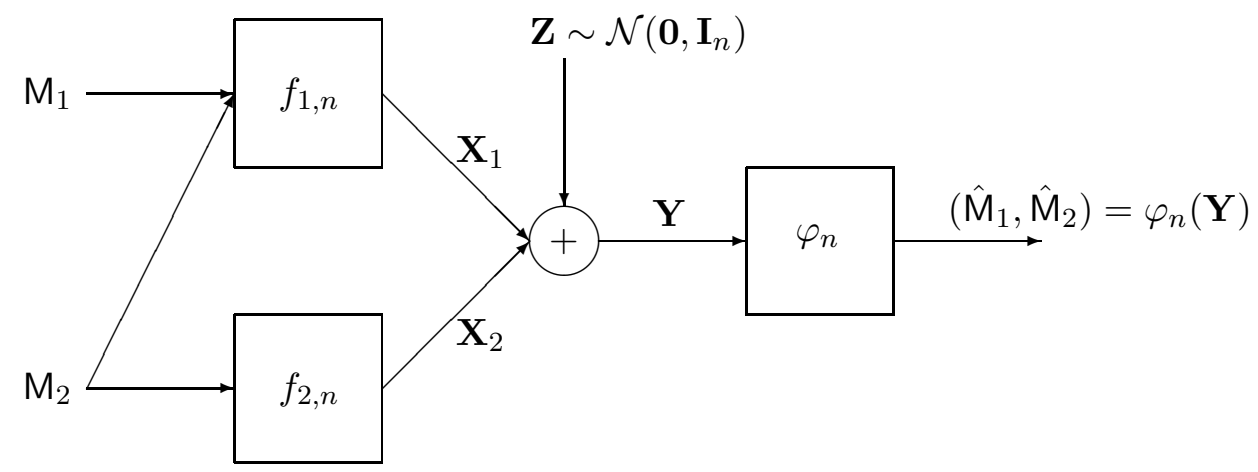

Fig. 1. The model for the Gaussian MAC with degraded message sets.

This study allows us to understand the fundamental tradeoffs between the rates of transmission and average error probability from a perspective different from the study of error exponents. Here, instead of fixing a pair of rates and studying the exponential decay of the error probability $\varepsilon$, we fix $\varepsilon$ and study the speed at which a sequence of rate pairs approaches an information-theoretic limit as the blocklength grows.

\section{A. Related Work}

The most notable early work on the second-order asymptotics for channel coding is that of Strassen [7], who considered discrete memoryless channels. For the single-user AWGN channel with a maximal power constraint $S$, a specialization of our model with $M_{2, n}=1$, Hayashi [4] and Polyanskiy et al. [8] showed that the optimum (highest) second-order coding rate is $\sqrt{\mathrm{V}(S)} \Phi^{-1}(\varepsilon)$, where $\mathrm{V}(x):=\frac{x(x+2)}{2(x+1)^{2}}$ is the Gaussian dispersion function. Polyanskiy et al. [8, Thm. 54] and Tan-Tomamichel [9] showed the refined asymptotic expansion

$$
\log M^{*}(n, \varepsilon)=n \mathrm{C}(S)+\sqrt{n \mathrm{~V}(S)} \Phi^{-1}(\varepsilon)+\frac{1}{2} \log n+O(1),
$$

where $M^{*}(n, \varepsilon)$ is the maximum size of a length- $n$ block code with average error probability not exceeding $\varepsilon$. In fact, the expression for $\mathrm{V}(S)$ was already known to Shannon [10, Sec. X], who analyzed the reliability function of the AWGN channel for rates close to capacity.

There have been numerous attempts to study the finite blocklength behavior and second-order asymptotics for MACs [11]-[19], but most of these works focus on inner bounds (the direct part). The development of tight and easily-evaluated converse bounds remains more modest, and those available do not match the direct part in general or are very restrictive (e.g. product channels were considered in [19]). We will see that the assumption of Gaussianity of the channel model together with the degradedness of the message sets allows us to circumvent some of the difficulties in proving second-order converses for the MAC, thus allowing us to obtain a conclusive second-order result.

We focus primarily on local second-order asymptotics propounded by Haim et al. [19] for general network information theory problems, where a boundary point is fixed and the rate of approach is characterized. This is different from the global asymptotics studied in [11]-[18], which we also study here as an initial step towards obtaining the local result.

\section{B. Main Contributions}

Our main contribution is the characterization of the set of admissible local second-order coding rates $\left(L_{1}, L_{2}\right)$ for points on the curved part of the boundary of the capacity region (Theorem 3). For a point characterized by $\rho \in(0,1)$, we show that the achievable second-order rate pairs $\left(L_{1}, L_{2}\right)$ are precisely those satisfying

$$
\left[\begin{array}{c}
L_{1} \\
L_{1}+L_{2}
\end{array}\right] \in \bigcup_{\beta \in \mathbb{R}}\left\{\beta \mathbf{D}(\rho)+\Psi^{-1}(\mathbf{V}(\rho), \varepsilon)\right\},
$$


where the entries of $\mathbf{D}(\rho)$ are the derivatives of the capacities in (1)-(2), $\mathbf{V}(\rho)$ is the dispersion matrix [11], [12], and $\Psi^{-1}$ is the 2-dimensional generalization of the inverse of the cumulative distribution function of a Gaussian. (All quantities are defined precisely in the sequel.) Thus, the contribution from the Gaussian approximation $\Psi^{-1}(\mathbf{V}(\rho), \varepsilon)$ is insufficient for characterizing the second-order asymptotics of multi-terminal channel coding problems in general; in this case, the vector $\mathbf{D}(\rho)$ is also required. This is in stark contrast to single-user problems (e.g. [3], [4], [6]-[8]) and the (two-encoder) Slepian-Wolf problem [5], [11] where the Gaussian approximation in terms of a dispersion quantity is sufficient for the second-order asymptotics. Our main result, which comprises the statement in (5), provides the first complete characterization of the local second-order asymptotics of a multi-user information theory problem in which the boundary of the capacity region (or optimal rate region for source coding problems) is curved.

Some intuition can be gained as to why the extra derivative term is needed by considering the possible angles of approach to a fixed boundary point $\left(R_{1}^{*}, R_{2}^{*}\right) \in \mathcal{C}$. Using a single multivariate Gaussian input distribution with correlation $\rho$ for all blocklengths is suboptimal in the second-order sense, as we can only achieve the angles of approach within the trapezoid parametrized by $\rho$ (see Fig. 2 and its caption). Our strategy is to consider sequences of input distributions that vary with the blocklength, i.e. they are parametrized by a sequence $\left\{\rho_{n}\right\}_{n \in \mathbb{N}}$ that converges to $\rho$ with speed $\Theta\left(\frac{1}{\sqrt{n}}\right)$. By a Taylor expansion of the first-order capacity vector $\mathbf{I}(\rho)$ (the vector of capacities in (1)-(2)),

$$
\mathbf{I}\left(\rho_{n}\right) \approx \mathbf{I}(\rho)+\left(\rho_{n}-\rho\right) \mathbf{D}(\rho)
$$

we see that this sequence results in the derivative/slope term $\mathbf{D}(\rho)$ observed in (5). Thus, the slope term corresponds to the deviation of $\rho_{n}$ from $\rho$, while the dispersion term involving $\mathbf{V}(\rho)$ results from, by now, standard central limit (fixed error) analysis of Shannon-theoretic coding problems [20].

We briefly comment on $\rho_{n}$ converging to $\rho$ at different speeds. If $\rho_{n}-\rho=o\left(\frac{1}{\sqrt{n}}\right)$, then the contribution of the remainder term in (6) is dominated by the dispersion term, and hence this is, up to second order, equivalent to considering $\rho_{n}=\rho$. In contrast, for $\rho_{n}-\rho=\omega\left(\frac{1}{\sqrt{n}}\right)$, this remainder term dominates the dispersion term. Nevertheless, this case does not feature in the local result, due to the way we define the second-order coding rate region in (3) - the backoff terms with coefficients $L_{1}$ and $L_{2}$ scale as $\sqrt{n}$. In particular, we show in the converse proof that if $\rho_{n}-\rho=\omega\left(\frac{1}{\sqrt{n}}\right)$, then no finite $\left(L_{1}, L_{2}\right)$ pairs satisfy the conditions in this definition.

An auxiliary contribution is a global second-order result [11], [19] (Theorem 2), which we use as an important stepping stone to obtain our local second-order result. We show that for any sequence $\rho_{n} \in[0,1]$, all rate pairs $\left(R_{1, n}, R_{2, n}\right)$ satisfying

$$
\left[\begin{array}{c}
R_{1, n} \\
R_{1, n}+R_{2, n}
\end{array}\right] \in \mathbf{I}\left(\rho_{n}\right)+\frac{\Psi^{-1}\left(\mathbf{V}\left(\rho_{n}\right), \varepsilon\right)}{\sqrt{n}}+o\left(\frac{1}{\sqrt{n}}\right) \mathbf{1}
$$

are achievable at blocklength $n$ and with average error probability no larger than $\varepsilon+o(1)$. Our proof technique yields a third-order term that remains $o\left(\frac{1}{\sqrt{n}}\right)$ no matter how $\rho_{n}$ varies with $n$. This property does not typically hold in previous results on multi-user fixed error asymptotics, but it turns out to be crucial in deriving the local result and the additional slope term (cf. (6)), at least using our proof techniques.

In summary, we submit that both the global and local results on their own provide complementary and useful insights into fundamental limits of the communication system, but in this paper our main goal is the latter.

\section{Problem Setting And Definitions}

In this section, we state the channel model, various definitions and some known results.

Notation: Given integers $l \leq m$, we use the discrete interval [1] notations $[l: m]:=\{l, \ldots, m\}$ and $[m]:=[1$ : $m]$. All $\log$ 's and exp's are with respect to the natural base e. The $\ell_{p}$-norm of the vectorized version of matrix $\mathbf{A}$ is denoted by $\|\mathbf{A}\|_{p}:=\left(\sum_{i, j}\left|a_{i, j}\right|^{p}\right)^{1 / p}$. For two vectors of the same length $\mathbf{a}, \mathbf{b} \in \mathbb{R}^{d}$, the notation $\mathbf{a} \leq \mathbf{b}$ means that $a_{j} \leq b_{j}$ for all $j \in[d]$. The notation $\mathcal{N}(\mathbf{u} ; \boldsymbol{\mu}, \boldsymbol{\Lambda})$ denotes the multivariate Gaussian probability density function (pdf) with mean $\boldsymbol{\mu}$ and covariance $\boldsymbol{\Lambda}$. The argument $\mathbf{u}$ will often be omitted. We use standard asymptotic notations: $f_{n} \in O\left(g_{n}\right)$ if and only if (iff) $\limsup _{n \rightarrow \infty}\left|f_{n} / g_{n}\right|<\infty ; f_{n} \in \Omega\left(g_{n}\right)$ iff $g_{n} \in O\left(f_{n}\right) ; f_{n} \in \Theta\left(g_{n}\right)$ iff $f_{n} \in O\left(g_{n}\right) \cap \Omega\left(g_{n}\right) ; f_{n} \in o\left(g_{n}\right)$ iff $\limsup _{n \rightarrow \infty}\left|f_{n} / g_{n}\right|=0$; and $f_{n} \in \omega\left(g_{n}\right)$ iff $\liminf _{n \rightarrow \infty}\left|f_{n} / g_{n}\right|=\infty$. 


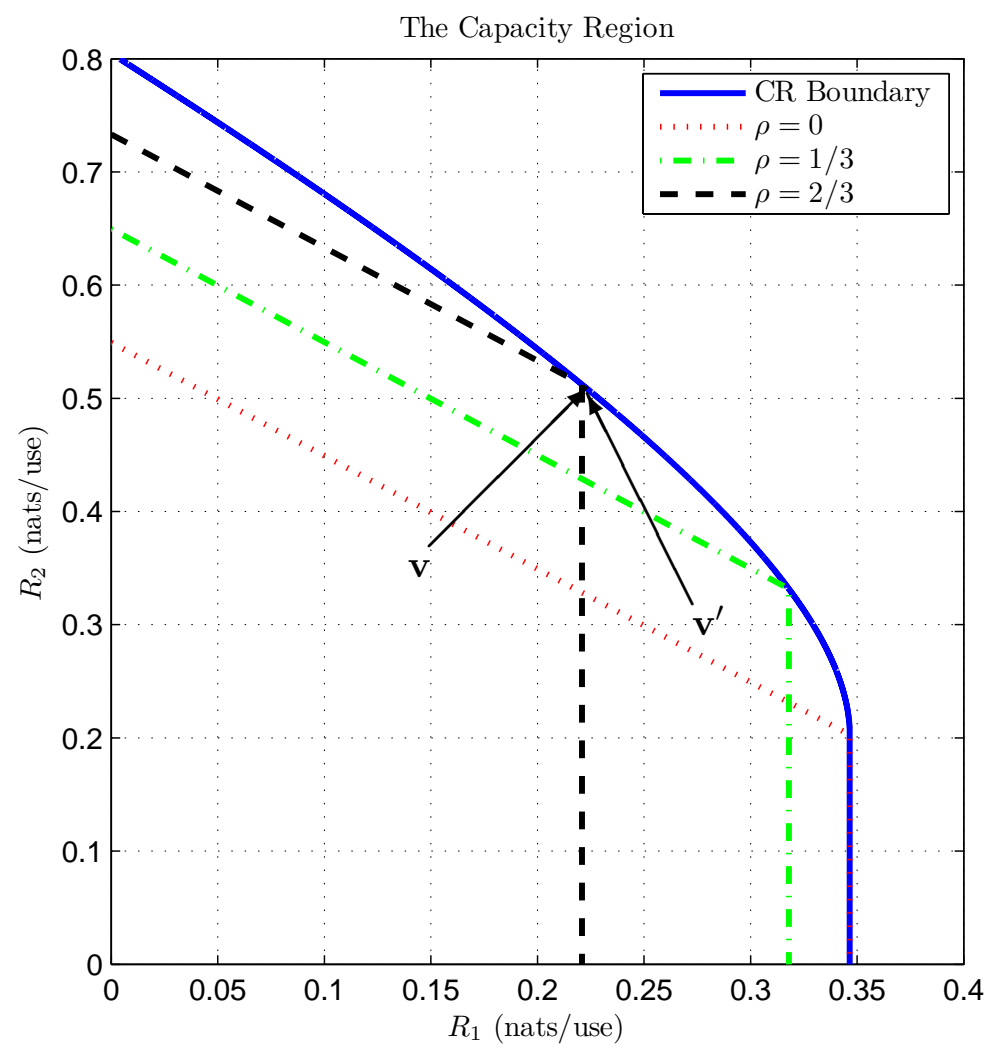

Fig. 2. Capacity region of the Gaussian MAC with degraded message sets in the case that $S_{1}=S_{2}=1$. Observe that $\rho \in[0,1]$ parametrizes points on the boundary. The vertical line segment corresponds to $\rho=0$, while the curved part corresponds to $\rho \in(0,1]$. Each $\rho \in(0,1]$ corresponds to a trapezoid of rate pairs that are achievable by a unique input distribution $\mathcal{N}(\mathbf{0}, \boldsymbol{\Sigma}(\rho))$. This coding strategy is insufficient to allow for all possible angles of approach to the fixed point parametrized by $\rho$, as there are non-empty regions within $\mathcal{C}$ that not in the trapezoid parametrized by $\rho$. In the figure above with $\rho=\frac{2}{3}$, one can approach the corner point in the direction indicated by the vector $\mathbf{v}$ using the fixed input distribution $\mathcal{N}\left(\mathbf{0}, \mathbf{\Sigma}\left(\frac{2}{3}\right)\right)$, but the same is not true of the direction indicated by $\mathbf{v}^{\prime}$, since the approach is from outside the trapezoid.

\section{A. Channel Model}

The signal model is given by

$$
Y=X_{1}+X_{2}+Z
$$

where $X_{1}$ and $X_{2}$ represent the inputs to the channel, $Z \sim \mathcal{N}(0,1)$ is additive Gaussian noise with mean zero and unit variance, and $Y$ is the output of the channel. Thus, the channel from $\left(X_{1}, X_{2}\right)$ to $Y$ can be written as

$$
W\left(y \mid x_{1}, x_{2}\right)=\frac{1}{\sqrt{2 \pi}} \exp \left(-\frac{1}{2}\left(y-x_{1}-x_{2}\right)^{2}\right) .
$$

The channel is used $n$ times in a memoryless manner without feedback. The channel inputs (i.e., the transmitted codewords) $\mathbf{x}_{1}=\left(x_{11}, \ldots, x_{1 n}\right)$ and $\mathbf{x}_{2}=\left(x_{21}, \ldots, x_{2 n}\right)$ are required to satisfy the maximal power constraints

$$
\left\|\mathbf{x}_{1}\right\|_{2}^{2} \leq n S_{1}, \quad \text { and } \quad\left\|\mathbf{x}_{2}\right\|_{2}^{2} \leq n S_{2},
$$

where $S_{1}$ and $S_{2}$ are arbitrary positive numbers. We do not incorporate multiplicative gains $g_{1}$ and $g_{2}$ to $X_{1}$ and $X_{2}$ in the channel model in (8); this is without loss of generality, since in the presence of these gains we may equivalently redefine (10) with $S_{j}^{\prime}:=S_{j} / g_{j}^{2}$ for $j=1,2$.

\section{B. Definitions}

Definition 1 (Code). An ( $\left.n, M_{1, n}, M_{2, n}, S_{1}, S_{2}, \varepsilon_{n}\right)$-code for the Gaussian MAC with degraded message sets consists of two encoders $f_{1, n}, f_{2, n}$ and a decoder $\varphi_{n}$ of the form $f_{1, n}:\left[M_{1, n}\right] \times\left[M_{2, n}\right] \rightarrow \mathbb{R}^{n}, f_{2, n}:\left[M_{2, n}\right] \rightarrow \mathbb{R}^{n}$ and 
$\varphi_{n}: \mathbb{R}^{n} \rightarrow\left[M_{1, n}\right] \times\left[M_{2, n}\right]$ satisfying

$$
\begin{aligned}
\left\|f_{1, n}\left(m_{1}, m_{2}\right)\right\|_{2}^{2} & \leq n S_{1} \quad \forall\left(m_{1}, m_{2}\right) \in\left[M_{1, n}\right] \times\left[M_{2, n}\right], \\
\left\|f_{2, n}\left(m_{2}\right)\right\|_{2}^{2} & \leq n S_{2} \quad \forall m_{2} \in\left[M_{2, n}\right], \\
\operatorname{Pr}\left(\left(\mathrm{M}_{1}, \mathrm{M}_{2}\right) \neq\left(\hat{\mathrm{M}}_{1}, \hat{\mathrm{M}}_{2}\right)\right) & \leq \varepsilon_{n},
\end{aligned}
$$

where the messages $\mathrm{M}_{1}$ and $\mathrm{M}_{2}$ are uniformly distributed on $\left[M_{1, n}\right]$ and $\left[M_{2, n}\right]$ respectively, and $\left(\hat{\mathrm{M}}_{1}, \hat{\mathrm{M}}_{2}\right):=$ $\varphi_{n}\left(Y^{n}\right)$ is the decoded message pair.

Since $S_{1}$ and $S_{2}$ are fixed positive numbers, we suppress the dependence of the subsequent definitions, results and parameters on these constants. We will often make reference to $(n, \varepsilon)$-codes; this is the family of ( $\left.n, M_{1, n}, M_{2, n}, S_{1}, S_{2}, \varepsilon\right)$-codes where the sizes $M_{1, n}, M_{2, n}$ are left unspecified.

Definition $2\left((n, \varepsilon)\right.$-Achievability). A pair of non-negative numbers $\left(R_{1}, R_{2}\right)$ is $(n, \varepsilon)$-achievable if there exists an $\left(n, M_{1, n}, M_{2, n}, S_{1}, S_{2}, \varepsilon_{n}\right)$-code such that

$$
\frac{1}{n} \log M_{j, n} \geq R_{j}, \quad j=1,2, \quad \text { and } \quad \varepsilon_{n} \leq \varepsilon .
$$

The $(n, \varepsilon)$-capacity region $\mathcal{C}(n, \varepsilon) \subset \mathbb{R}_{+}^{2}$ is defined to be the set of all $(n, \varepsilon)$-achievable rate pairs $\left(R_{1}, R_{2}\right)$.

Definition 2 is a non-asymptotic one that is used primarily for the global second-order results. We now introduce asymptotic-type definitions that involve the existence of sequences of codes.

Definition 3 (First-Order Coding Rates). A pair of non-negative numbers $\left(R_{1}, R_{2}\right)$ is $\varepsilon$-achievable if there exists a sequence of $\left(n, M_{1, n}, M_{2, n}, S_{1}, S_{2}, \varepsilon_{n}\right)$-codes such that

$$
\liminf _{n \rightarrow \infty} \frac{1}{n} \log M_{j, n} \geq R_{j}, \quad j=1,2, \quad \text { and } \quad \limsup _{n \rightarrow \infty} \varepsilon_{n} \leq \varepsilon .
$$

The $\varepsilon$-capacity region $\mathcal{C}(\varepsilon) \subset \mathbb{R}_{+}^{2}$ is defined to be the closure of the set of all $\varepsilon$-achievable rate pairs $\left(R_{1}, R_{2}\right)$. The capacity region $\mathcal{C}$ is defined as

$$
\mathcal{C}:=\bigcap_{\varepsilon>0} \mathcal{C}(\varepsilon)=\lim _{\varepsilon \rightarrow 0} \mathcal{C}(\varepsilon)
$$

where the limit exists because of the monotonicity of $\mathcal{C}(\varepsilon)$.

Next, we state the most important definitions concerning local second-order coding rates in the spirit of NomuraHan [5] and Tan-Kosut [11]. We will spend the majority of the paper developing tools to characterize these rates. Here $\left(R_{1}^{*}, R_{2}^{*}\right)$ is a pair of rates on the boundary of $\mathcal{C}(\varepsilon)$.

Definition 4 (Second-Order Coding Rates). A pair of numbers $\left(L_{1}, L_{2}\right)$ is $\left(\varepsilon, R_{1}^{*}, R_{2}^{*}\right)$-second-order achievable if there exists a sequence of $\left(n, M_{1, n}, M_{2, n}, S_{1}, S_{2}, \varepsilon_{n}\right)$-codes such that

$$
\liminf _{n \rightarrow \infty} \frac{1}{\sqrt{n}}\left(\log M_{j, n}-n R_{j}^{*}\right) \geq L_{j}, \quad j=1,2, \quad \text { and } \quad \limsup _{n \rightarrow \infty} \varepsilon_{n} \leq \varepsilon
$$

The $\left(\varepsilon, R_{1}^{*}, R_{2}^{*}\right)$-optimal second-order coding rate region $\mathcal{L}\left(\varepsilon ; R_{1}^{*}, R_{2}^{*}\right) \subset \mathbb{R}^{2}$ is defined to be the closure of the set of all $\left(\varepsilon, R_{1}^{*}, R_{2}^{*}\right)$-second-order achievable rate pairs $\left(L_{1}, L_{2}\right)$.

Stated differently, if $\left(L_{1}, L_{2}\right)$ is $\left(\varepsilon, R_{1}^{*}, R_{2}^{*}\right)$-second-order achievable, then there are codes whose error probabilities are asymptotically no larger than $\varepsilon$, and whose sizes $\left(M_{1, n}, M_{2, n}\right)$ satisfy the asymptotic relation in (3). Even though we refer to $L_{1}$ and $L_{2}$ as "rates", they may be negative [3]-[6]. A negative value corresponds to a backoff from the first-order term, whereas a positive value corresponds to an addition to the first-order term. 


\section{Existing First-Order Results}

To put things in context, we review some existing results concerning the $\varepsilon$-capacity region. To state the result compactly, we define the mutual information (or capacity) vector as

$$
\mathbf{I}(\rho)=\left[\begin{array}{c}
I_{1}(\rho) \\
I_{12}(\rho)
\end{array}\right]:=\left[\begin{array}{c}
\mathrm{C}\left(S_{1}\left(1-\rho^{2}\right)\right) \\
\mathrm{C}\left(S_{1}+S_{2}+2 \rho \sqrt{S_{1} S_{2}}\right)
\end{array}\right]
$$

where $\rho \in[-1,1]$. For a pair of rates $\left(R_{1}, R_{2}\right)$, let the rate vector be

$$
\mathbf{R}:=\left[\begin{array}{c}
R_{1} \\
R_{1}+R_{2}
\end{array}\right] .
$$

A statement of the following result is provided in [1, Ex. 5.18(b)]. A weak converse was proved for the more general Gaussian MAC a with common message in [21].

Proposition 1 (Capacity Region). The capacity region of the Gaussian MAC with degraded message sets is given by

$$
\mathcal{C}=\bigcup_{0 \leq \rho \leq 1}\left\{\left(R_{1}, R_{2}\right) \in \mathbb{R}_{+}^{2}: \mathbf{R} \leq \mathbf{I}(\rho)\right\}
$$

The union on the right is a subset of $\mathcal{C}(\varepsilon)$ for every $\varepsilon \in(0,1)$. However, only the weak converse is implied by (20). The strong converse has not been demonstrated previously. Thus, a by-product of the derivation of the second-order asymptotics in this paper is the strong converse, allowing us to assert that for all $\varepsilon \in(0,1)$,

$$
\mathcal{C}=\mathcal{C}(\varepsilon)
$$

The direct part of Proposition 1 can be proved using superposition coding [2], treating $X_{2}$ as the cloud center and $X_{1}$ as the satellite codeword. The input distribution to achieve a point on the boundary characterized by some $\rho \in[0,1]$ is a 2 -dimensional Gaussian with mean zero and covariance matrix

$$
\boldsymbol{\Sigma}(\rho):=\left[\begin{array}{cc}
S_{1} & \rho \sqrt{S_{1} S_{2}} \\
\rho \sqrt{S_{1} S_{2}} & S_{2}
\end{array}\right] .
$$

Thus, the parameter $\rho$ represents the correlation between the two users' codewords.

\section{GlobAl SeCOND-ORder Results}

In this section, we present inner and outer bounds on $\mathcal{C}(n, \varepsilon)$. We begin with some definitions. Let $\mathrm{V}(x, y):=$ $\frac{x(y+2)}{2(x+1)(y+1)}$ be the Gaussian cross-dispersion function and let $\mathrm{V}(x):=\mathrm{V}(x, x)$ be the Gaussian dispersion function [4], [8], [10] for a single-user AWGN channel with signal-to-noise ratio $x$. For fixed $0 \leq \rho \leq 1$, define the information-dispersion matrix

$$
\mathbf{V}(\rho):=\left[\begin{array}{cc}
V_{1}(\rho) & V_{1,12}(\rho) \\
V_{1,12}(\rho) & V_{12}(\rho)
\end{array}\right]
$$

where the elements of the matrix are

$$
\begin{aligned}
V_{1}(\rho) & :=\mathrm{V}\left(S_{1}\left(1-\rho^{2}\right)\right), \\
V_{1,12}(\rho) & :=\mathrm{V}\left(S_{1}\left(1-\rho^{2}\right), S_{1}+S_{2}+2 \rho \sqrt{S_{1} S_{2}}\right), \\
V_{12}(\rho) & :=\mathrm{V}\left(S_{1}+S_{2}+2 \rho \sqrt{S_{1} S_{2}}\right) .
\end{aligned}
$$

Let $\left(X_{1}, X_{2}\right) \sim P_{X_{1}, X_{2}}=\mathcal{N}(\mathbf{0} ; \boldsymbol{\Sigma}(\rho))$, and define $Q_{Y \mid X_{2}}$ and $Q_{Y}$ to be Gaussian distributions induced by $P_{X_{1}, X_{2}}$ and the channel $W$, namely

$$
\begin{aligned}
Q_{Y \mid X_{2}}\left(y \mid x_{2}\right) & :=\mathcal{N}\left(y ; x_{2}\left(1+\rho \sqrt{S_{1} / S_{2}}\right), 1+S_{1}\left(1-\rho^{2}\right)\right), \\
Q_{Y}(y) & :=\mathcal{N}\left(y ; 0,1+S_{1}+S_{2}+2 \rho \sqrt{S_{1} S_{2}}\right)
\end{aligned}
$$



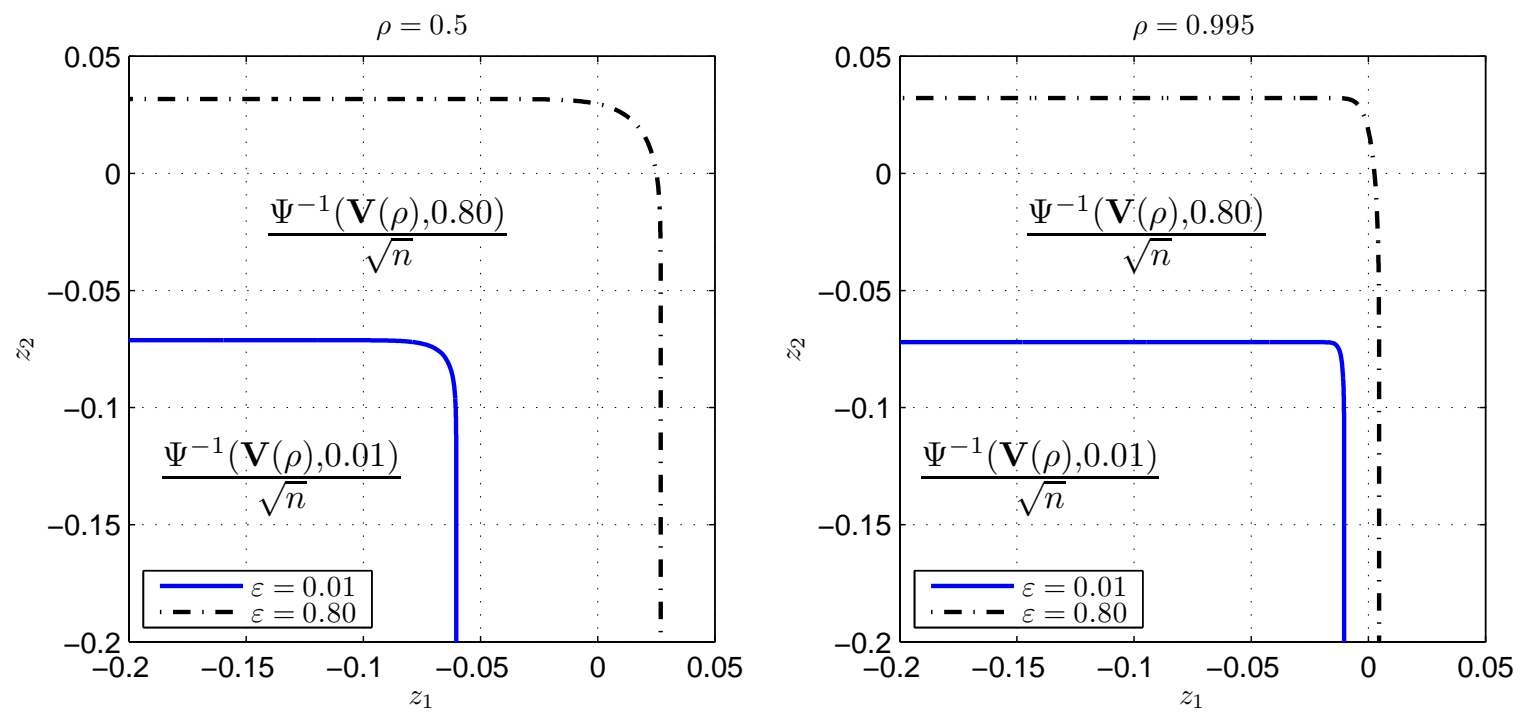

Fig. 3. Illustration of the set $\Psi^{-1}(\mathbf{V}(\rho), \varepsilon) / \sqrt{n}$ with $n=500, S_{1}=S_{2}=1, \rho=0.5$ (moderate correlation) and $\rho=0.995$ (high correlation). The information dispersion matrix $\mathbf{V}(\rho)$ is defined in (23). In the plots, $\varepsilon$ takes two values, 0.01 and 0.80 .

It should be noted that the random variables $\left(X_{1}, X_{2}\right)$ and the densities $Q_{Y \mid X_{2}}$ and $Q_{Y}$ all depend on $\rho$; this dependence is suppressed throughout the paper. The mutual information vector $\mathbf{I}(\rho)$ and information-dispersion matrix $\mathbf{V}(\rho)$ are the mean vector and conditional covariance matrix of the information density vector

$$
\mathbf{j}\left(X_{1}, X_{2}, Y\right):=\left[\begin{array}{c}
j_{1}\left(X_{1}, X_{2}, Y\right) \\
j_{12}\left(X_{1}, X_{2}, Y\right)
\end{array}\right]=\left[\log \frac{W\left(Y \mid X_{1}, X_{2}\right)}{Q_{Y \mid X_{2}}\left(Y \mid X_{2}\right)}, \quad \log \frac{W\left(Y \mid X_{1}, X_{2}\right)}{Q_{Y}(Y)}\right]^{T} .
$$

That is, we can write $\mathbf{I}(\rho)$ and $\mathbf{V}(\rho)$ as

$$
\begin{aligned}
\mathbf{I}(\rho) & =\mathbb{E}\left[\mathbf{j}\left(X_{1}, X_{2}, Y\right)\right], \\
\mathbf{V}(\rho) & =\mathbb{E}\left[\operatorname{Cov}\left(\mathbf{j}\left(X_{1}, X_{2}, Y\right) \mid X_{1}, X_{2}\right)\right] .
\end{aligned}
$$

For a given point $\left(z_{1}, z_{2}\right) \in \mathbb{R}^{2}$ and a (non-zero) positive semi-definite matrix $\mathbf{V}$, define

$$
\Psi\left(z_{1}, z_{2} ; \mathbf{V}\right):=\int_{-\infty}^{z_{2}} \int_{-\infty}^{z_{1}} \mathcal{N}(\mathbf{u} ; \mathbf{0}, \mathbf{V}) \mathrm{d} \mathbf{u}
$$

and for a given $\varepsilon \in(0,1)$, define the set

$$
\Psi^{-1}(\mathbf{V}, \varepsilon):=\left\{\left(z_{1}, z_{2}\right) \in \mathbb{R}^{2}: \Psi\left(-z_{1},-z_{2} ; \mathbf{V}\right) \geq 1-\varepsilon\right\} .
$$

These quantities can be thought of as the generalization of the cumulative distribution function (cdf) of the standard Gaussian $\Phi(z):=\int_{-\infty}^{z} \mathcal{N}(u ; 0,1) \mathrm{d} u$ and its inverse $\Phi^{-1}(\varepsilon):=\sup \{z \in \mathbb{R}: \Phi(-z) \geq 1-\varepsilon\}$ to the bivariate case. For $\varepsilon<\frac{1}{2}$, the points contained in $\Psi^{-1}(\mathbf{V}, \varepsilon)$ have negative coordinates. See Fig. 3 for an illustration of (scaled versions of) $\Psi^{-1}(\mathbf{V}(\rho), \varepsilon)$.

Let $\underline{g}(\rho, \varepsilon, n)$ and $\bar{g}(\rho, \varepsilon, n)$ be arbitrary functions of $\rho, \varepsilon$ and $n$ for now, and define the inner and outer regions

$$
\begin{array}{r}
\mathcal{R}_{\text {in }}(n, \varepsilon ; \rho):=\left\{\left(R_{1}, R_{2}\right) \in \mathbb{R}^{2}: \mathbf{R} \in \mathbf{I}(\rho)+\frac{\Psi^{-1}(\mathbf{V}(\rho), \varepsilon)}{\sqrt{n}}+\underline{g}(\rho, \varepsilon, n) \mathbf{1}\right\}, \\
\mathcal{R}_{\text {out }}(n, \varepsilon ; \rho):=\left\{\left(R_{1}, R_{2}\right) \in \mathbb{R}^{2}: \mathbf{R} \in \mathbf{I}(\rho)+\frac{\Psi^{-1}(\mathbf{V}(\rho), \varepsilon)}{\sqrt{n}}+\bar{g}(\rho, \varepsilon, n) \mathbf{1}\right\} .
\end{array}
$$

Theorem 2 (Global Bounds on the $(n, \varepsilon)$-Capacity Region). There exist functions $\underline{g}(\rho, \varepsilon, n)$ and $\bar{g}(\rho, \varepsilon, n)$ such that the $(n, \varepsilon)$-capacity region satisfies

$$
\bigcup_{0 \leq \rho \leq 1} \mathcal{R}_{\text {in }}(n, \varepsilon ; \rho) \subset \mathcal{C}(n, \varepsilon) \subset \bigcup_{-1 \leq \rho \leq 1} \mathcal{R}_{\text {out }}(n, \varepsilon ; \rho),
$$


and such that $\underline{g}$ and $\bar{g}$ satisfy the following properties:

1) For any $\varepsilon \in(0,1)$ and any sequence $\left\{\rho_{n}\right\}$ converging to some value $\rho \notin\{-1,+1\}$, we have

$$
\underline{g}\left(\rho_{n}, \varepsilon, n\right)=O\left(\frac{\log n}{n}\right), \quad \text { and } \quad \bar{g}\left(\rho_{n}, \varepsilon, n\right)=O\left(\frac{\log n}{n}\right) .
$$

2) For any $\varepsilon \in(0,1)$ and any sequence $\left\{\rho_{n}\right\}$ with $\rho_{n} \rightarrow \rho \in\{-1,+1\}$, we have

$$
\underline{g}\left(\rho_{n}, \varepsilon, n\right)=o\left(\frac{1}{\sqrt{n}}\right), \quad \text { and } \quad \bar{g}\left(\rho_{n}, \varepsilon, n\right)=o\left(\frac{1}{\sqrt{n}}\right) .
$$

The proof of Theorem 2 is provided in Section VI. We remark that even though the union for the outer bound is taken over $\rho \in[-1,1]$, only the values $\rho \in[0,1]$ will play a role in establishing the local asymptotics in Section IV since negative values of $\rho$ are not even first-order optimal, i.e. they fail to achieve a point on the boundary of the capacity region.

Note that we do not claim the remainder terms in (37)-(38) to be uniform in $\left\{\rho_{n}\right\}$; such uniformity will not be required in establishing our main local result below. On the other hand, it is crucial that values of $\rho$ varying with $n$ are handled (in contrast, most existing global results in other settings consider fixed input distributions).

\section{LOCAL SECOND-ORder CODING RATES}

In this section, we present our main result, namely, the characterization of the $\left(\varepsilon, R_{1}^{*}, R_{2}^{*}\right)$-optimal second-order coding rate region $\mathcal{L}\left(\varepsilon ; R_{1}^{*}, R_{2}^{*}\right)$ (see Definition 4), where $\left(R_{1}^{*}, R_{2}^{*}\right)$ is an arbitrary point on the boundary of $\mathcal{C}$. Our result is stated in terms of the derivative of the mutual information vector with respect to $\rho$, namely

$$
\mathbf{D}(\rho)=\left[\begin{array}{c}
D_{1}(\rho) \\
D_{12}(\rho)
\end{array}\right]:=\frac{\mathrm{d}}{\mathrm{d} \rho}\left[\begin{array}{c}
I_{1}(\rho) \\
I_{12}(\rho)
\end{array}\right],
$$

where the individual derivatives are given by

$$
\begin{aligned}
\frac{\mathrm{d} I_{1}(\rho)}{\mathrm{d} \rho} & =\frac{-S_{1} \rho}{1+S_{1}\left(1-\rho^{2}\right)}, \\
\frac{\mathrm{d} I_{12}(\rho)}{\mathrm{d} \rho} & =\frac{\sqrt{S_{1} S_{2}}}{1+S_{1}+S_{2}+2 \rho \sqrt{S_{1} S_{2}}} .
\end{aligned}
$$

For a vector $\mathbf{v}=\left(v_{1}, v_{2}\right) \in \mathbb{R}^{2}$, we define the down-set of $\mathbf{v}$ as

$$
\mathbf{v}^{-}:=\left\{\left(w_{1}, w_{2}\right) \in \mathbb{R}^{2}: w_{1} \leq v_{1}, w_{2} \leq v_{2}\right\} .
$$

Theorem 3 (Optimal Second-Order Coding Rate Region). Depending on $\left(R_{1}^{*}, R_{2}^{*}\right)$, we have the following three cases:

(i) If $R_{1}^{*}=I_{1}(0)$ and $R_{1}^{*}+R_{2}^{*} \leq I_{12}(0)$ (vertical segment of the boundary corresponding to $\rho=0$ ), then

$$
\mathcal{L}\left(\varepsilon ; R_{1}^{*}, R_{2}^{*}\right)=\left\{\left(L_{1}, L_{2}\right) \in \mathbb{R}^{2}: L_{1} \leq \sqrt{V_{1}(0)} \Phi^{-1}(\varepsilon)\right\} .
$$

(ii) If $R_{1}^{*}=I_{1}(\rho)$ and $R_{1}^{*}+R_{2}^{*}=I_{12}(\rho)$ (curved segment of the boundary corresponding to $0<\rho<1$ ), then

$$
\mathcal{L}\left(\varepsilon ; R_{1}^{*}, R_{2}^{*}\right)=\left\{\left(L_{1}, L_{2}\right) \in \mathbb{R}^{2}:\left[\begin{array}{c}
L_{1} \\
L_{1}+L_{2}
\end{array}\right] \in \bigcup_{\beta \in \mathbb{R}}\left\{\beta \mathbf{D}(\rho)+\Psi^{-1}(\mathbf{V}(\rho), \varepsilon)\right\}\right\} .
$$

(iii) If $R_{1}^{*}=0$ and $R_{1}^{*}+R_{2}^{*}=I_{12}(1)$ (point on the vertical axis corresponding to $\rho=1$ ), then

$$
\mathcal{L}\left(\varepsilon ; R_{1}^{*}, R_{2}^{*}\right)=\left\{\left(L_{1}, L_{2}\right) \in \mathbb{R}^{2}:\left[\begin{array}{c}
L_{1} \\
L_{1}+L_{2}
\end{array}\right] \in \bigcup_{\beta \leq 0}\left\{\beta \mathbf{D}(1)+\left[\sqrt{V_{12}(1)} \Phi^{-1}(\varepsilon)\right]^{-}\right\}\right\} .
$$

The proof of Theorem 3 is provided in Section VII It leverages on the global second-order result in Theorem 2 


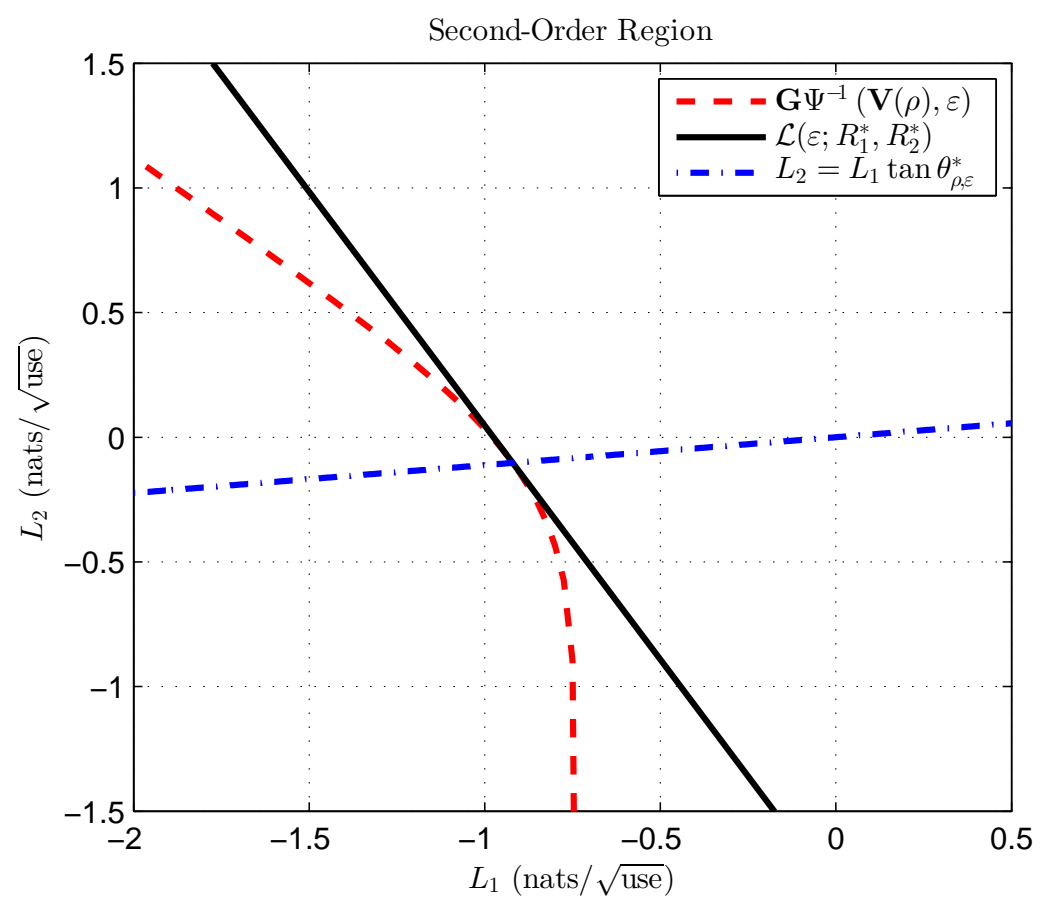

Fig. 4. Second-order coding rates in nats $\sqrt{\text { use }}$ with $S_{1}=S_{2}=1, \rho=\frac{1}{2}$ and $\varepsilon=0.1$. The regions $\mathbf{G} \Psi^{-1}(\mathbf{V}(\rho), \varepsilon)($ with $\mathbf{G}:=$ $[1,0 ;-1,1])$ and $\mathcal{L}\left(\varepsilon ; R_{1}^{*}, R_{2}^{*}\right)$ are to the bottom left of the boundaries. We also plot the line $L_{2}=L_{1} \tan \theta_{\rho, \varepsilon}^{*}$, where $\theta_{\rho, \varepsilon}^{*}$ is the unique angle $\theta$ for which the intersection of the boundary of $\mathcal{L}\left(\varepsilon ; R_{1}^{*}, R_{2}^{*}\right)$ and the line $L_{2}=L_{1} \tan \theta$ coincides with the boundary of $\mathbf{G} \Psi^{-1}(\mathbf{V}(\rho), \varepsilon)$.

\section{A. Discussion}

Observe that in case (i), the second-order region is simply characterized by a scalar dispersion term $V_{1}(0)$ and the inverse of the Gaussian cdf $\Phi^{-1}$. Roughly speaking, in this part of the boundary, there is effectively only a single rate constraint in terms of $R_{1}$, since we are operating "far away" from the sum rate constraint. This results in a large deviations-type event for the sum rate constraint which has no bearing on second-order asymptotics; see further discussions in [5], [11] and [19].

Cases (ii)-(iii) are more interesting, and their proofs are non-trivial. As in Nomura-Han [5] and Tan-Kosut [11], the second-order asymptotics for case (ii) depend on the dispersion matrix $\mathbf{V}(\rho)$ and the 2-dimensional analogue of the inverse of the Gaussian cdf $\Psi^{-1}$. However, in our setting, the expression containing $\Psi^{-1}$ alone (i.e. the expression obtained by setting $\beta=0$ in (44)) corresponds to only considering the unique input distribution $\mathcal{N}(\mathbf{0}, \boldsymbol{\Sigma}(\rho))$ achieving the point $\left(R_{1}^{*}, R_{2}^{*}\right)=\left(I_{1}(\rho), I_{12}(\rho)-I_{1}(\rho)\right)$. As discussed in the introduction and the caption of Fig. 2 this is not sufficient to achieve all second-order coding rates, since there are non-empty regions within the capacity region that are not contained in the trapezoid of rate pairs achievable using $\mathcal{N}(\mathbf{0}, \boldsymbol{\Sigma}(\rho))$. Using a sequence of input distributions parametrized by $\rho_{n}$ converging to $\rho$ with rate $\Theta\left(\frac{1}{\sqrt{n}}\right)$, we obtain the Taylor expansion in (6), yielding the gradient term $\mathbf{D}(\rho)$.

For the converse, we consider an arbitrary sequence of codes with rate pairs $\left\{\left(R_{1, n}, R_{2, n}\right)\right\}_{n \in \mathbb{N}}$ converging to $\left(I_{1}(\rho), I_{12}(\rho)-I_{1}(\rho)\right)$ with second-order behavior given by (17). From the global result, we know $\left[R_{1, n}, R_{1, n}+\right.$ $\left.R_{2, n}\right]^{T} \in \mathcal{R}_{\text {out }}\left(n, \varepsilon ; \rho_{n}\right)$ for some sequence $\left\{\rho_{n}\right\}$. Combining this with the definition of the second-order coding rate in (17), we establish that $\rho_{n} \rightarrow \rho$. The final result readily follows provided that $\rho_{n}=\rho+O\left(\frac{1}{\sqrt{n}}\right)$, and the remaining cases are shown to have no effect on $\mathcal{L}$.

A similar discussion holds true for case (iii); the main differences are that the covariance matrix is singular, and that the union in (45) is taken over $\beta \leq 0$ only, since $\rho_{n}$ can only approach one from below.

\section{B. Second-Order Asymptotics for a Given Angle of Approach}

Here we study the second-order behavior when a point on the boundary is approached from a given angle, as was done in Tan-Kosut [11]. We focus on the most interesting case in Theorem 3, namely, case (ii) corresponding 


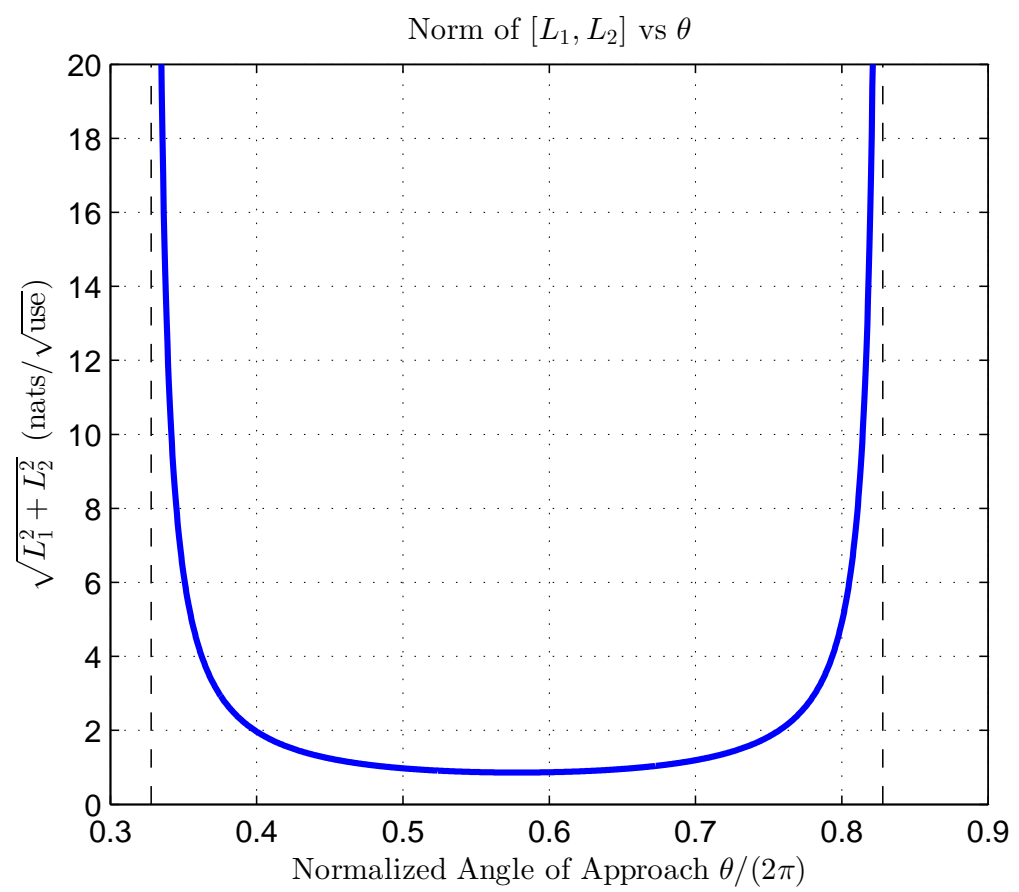

Fig. 5. Plot of $\sqrt{L_{1}^{2}+L_{2}^{2}}$ against the angle of approach $\theta$ with the same parameters as in Fig. 4 The second-order rates $L_{1}, L_{2}$, as functions of $\theta$, are defined in 48 . Here, $\pi+\arctan \left(a_{\rho}\right) \approx 0.328 \times 2 \pi$ and $2 \pi+\arctan \left(a_{\rho}\right) \approx 0.828 \times 2 \pi$ are the critical angles where $\sqrt{L_{1}^{2}+L_{2}^{2}}$ diverges.

to $\rho \in(0,1)$. Case (iii) can be handled similarly, and in case (i) the angle of approach is of little interest, since $L_{2}$ can be arbitrary.

First, we present an alternative expression for the set $\mathcal{L}=\mathcal{L}\left(\varepsilon ; R_{1}^{*}, R_{2}^{*}\right)$ given in (44) with $R_{1}^{*}=I_{1}(\rho)$ and $R_{1}^{*}+R_{2}^{*}=I_{12}(\rho)$ for some $\rho \in(0,1)$. It is easily seen that $\left(L_{1}, L_{2}\right) \in \mathcal{L}$ implies $\left(L_{1}+\beta D_{1}(\rho), L_{2}+\beta D_{2}(\rho)\right) \in \mathcal{L}$, where $D_{2}(\rho):=D_{12}(\rho)-D_{1}(\rho)$. It follows that $\mathcal{L}$ equals the set of all points lying below a straight line with slope $\frac{D_{2}(\rho)}{D_{1}(\rho)}$ which intersects the boundary of $\mathbf{G} \Psi^{-1}(\mathbf{V}(\rho), \varepsilon)$, where $\mathbf{G}:=[1,0 ;-1,1]$ is the invertible matrix that transforms the coordinate system from $\left[L_{1}, L_{1}+L_{2}\right]^{T}$ to $\left[L_{1}, L_{2}\right]^{T}$. (In other words, $\mathbf{G} \Psi^{-1}(\mathbf{V}(\rho), \varepsilon)$ is as in (44)), but with the union removed and $\beta$ set to 0 .) In light of the preceding discussion,

$$
\mathcal{L}\left(\varepsilon ; R_{1}^{*}, R_{2}^{*}\right)=\left\{\left(L_{1}, L_{2}\right): L_{2} \leq a_{\rho} L_{1}+b_{\rho, \varepsilon}\right\}
$$

where

$$
a_{\rho}:=\frac{D_{2}(\rho)}{D_{1}(\rho)}, \quad \text { and } \quad b_{\rho, \varepsilon}:=\inf \left\{b:\left(L_{1}, a_{\rho} L_{1}+b\right) \in \mathbf{G} \Psi^{-1}(\mathbf{V}(\rho), \varepsilon) \text { for some } L_{1} \in \mathbb{R}\right\} .
$$

We provide an example in Fig. 4 with the parameters $S_{1}=S_{2}=1, \rho=\frac{1}{2}$ and $\varepsilon=0.1$. Since $\varepsilon<\frac{1}{2}$, the boundary point $\left(R_{1}^{*}, R_{2}^{*}\right)$ is approached from the inside (see Fig. 3, where for $\varepsilon<\frac{1}{2}$, the set $\Psi^{-1}(\mathbf{V}, \varepsilon)$ only contains points with negative coordinates).

Given the gradient $a_{\rho}$, the offset $b_{\rho, \varepsilon}$, and an angle $\theta$ (measured with respect to the horizontal axis), we seek the pair $\left(L_{1}, L_{2}\right)$ on the boundary of $\mathcal{L}\left(\varepsilon ; R_{1}^{*}, R_{2}^{*}\right)$ such that $L_{2}=L_{1} \tan \theta$. It is easily seen that this point is obtained by solving for the intersection of the line $L_{2}=a_{\rho} L_{1}+b_{\rho, \varepsilon}$ with $L_{2}=L_{1} \tan \theta$. The two lines coincide when

$$
L_{1}=\frac{b_{\rho, \varepsilon}}{\tan \theta-a_{\rho}}, \quad \text { and } \quad L_{2}=\frac{b_{\rho, \varepsilon} \tan \theta}{\tan \theta-a_{\rho}} .
$$

In Fig. 4, we see that there is only a single angle $\theta_{\rho, \varepsilon}^{*} \approx 3.253$ rads for which the point of intersection in (48) is also on the boundary of $\mathbf{G} \Psi^{-1}(\mathbf{V}(\rho), \varepsilon)$, yielding $\left(L_{1}, L_{2}\right) \approx(-0.920,-0.103)$. In other words, there is only one angle for which coding with a fixed input distribution $\mathcal{N}(\mathbf{0}, \mathbf{V}(\rho))$ is optimal in the second-order sense (i.e. for 
which the added term $\beta \mathbf{D}(\rho)$ in (44) is of no additional help and $\beta=0$ is optimal). For all the other angles, we should choose a non-zero coefficient $\beta$, which corresponds to choosing an input distribution that varies with $n$.

Finally, in Fig. 5, we plot the norm of the vector of second-order rates $\left[L_{1}, L_{2}\right]^{T}$ in (48) against $\theta$, the angle of approach. For $\varepsilon<\frac{1}{2}$, the point $\left[L_{1}, L_{2}\right]^{T}$ may be interpreted as that corresponding to the "smallest backoff" from the first-order optimal rates 11 Thus, $\sqrt{L_{1}^{2}+L_{2}^{2}}$ is a measure of the total backoff. For $\varepsilon>\frac{1}{2},\left[L_{1}, L_{2}\right]^{T}$ corresponds to the "largest addition" to the first-order rates. It is noted that the norm tends to infinity when the angle tends to $\pi+\arctan \left(a_{\rho}\right)$ (from above) or $2 \pi+\arctan \left(a_{\rho}\right)$ (from below). This corresponds to an approach almost parallel to the gradient at the point on the boundary parametrized by $\rho$. A similar phenomenon was observed for the Slepian-Wolf problem [11].

\section{Concluding Remarks}

We have identified the optimal second-order coding rate region of the Gaussian MAC with degraded message sets. There are two reasons as to why the analysis here is more tractable vis-à-vis finite blocklength or second-order analysis for the the discrete memoryless MAC (DM-MAC) studied extensively in [11]-[13], [17]-[19]. Gaussianity allows us to identify the boundary of the capacity region and associate each point on the boundary with an input distribution parametrized by $\rho$. For the DM-MAC, one needs to take the convex closure of the union over input distributions $P_{X_{1}, X_{2}}$ to define the capacity region [1, Sec. 4.5], and hence the boundary points are more difficult to characterize. In addition, one needs to ensure in a converse proof (possibly related to the wringing technique of Ahlswede [22]) that the codewords pairs are almost orthogonal. By leveraging on the assumption of degraded message sets, we circumvent this requirement.

For future investigations, we note that the Gaussian broadcast channel [1, Sec. 5.5] is a problem which is similar to the Gaussian MAC with degraded message sets (e.g. both require superposition coding, and each point on the boundary is achieved by a unique input distribution). As such, we expect that some of the second-order analysis techniques contained herein may be applicable to the Gaussian broadcast channel. The authors have recently adapted the techniques herein for the discrete memoryless MAC with degraded message sets [23], again obtaining a conclusive characterization of the second-order rate region.

\section{Proof of Theorem 2: Global Second-Order Result}

\section{A. Converse Part}

We first prove the outer bound in (36). The analysis is split into seven steps.

1) A Reduction from Maximal to Equal Power Constraints: Let $\mathcal{C}_{\text {eq }}(n, \varepsilon)$ be the $(n, \varepsilon)$-capacity region in the case that (11) and (12) are equality constraints, i.e., $\left\|f_{1, n}\left(m_{1}, m_{2}\right)\right\|_{2}^{2}=n S_{1}$ and $\left\|f_{2, n}\left(m_{2}\right)\right\|_{2}^{2}=n S_{2}$ for all $\left(m_{1}, m_{2}\right)$. We claim that

$$
\mathcal{C}_{\text {eq }}(n, \varepsilon) \subset \mathcal{C}(n, \varepsilon) \subset \mathcal{C}_{\text {eq }}(n+1, \varepsilon) .
$$

The lower bound is obvious, because the equal power constraint is more stringent than the maximal power constraint. The upper bound follows by noting that the decoder for the length- $(n+1)$ code can ignore the last symbol, which can be chosen to equalize the powers.

It follows from (49) that for the purpose of second-order asymptotics, $\mathcal{C}_{\text {eq }}(n, \varepsilon)$ and $\mathcal{C}(n, \varepsilon)$ are equivalent. This argument was also used in [8, Lem. 39] and [10, Sec. XIII]. Henceforth, we assume that all codewords $\left(\mathbf{x}_{1}, \mathbf{x}_{2}\right)$ have normalized powers exactly equal to $\left(S_{1}, S_{2}\right)$.

2) A Reduction from Average to Maximal Error Probability: Let $\mathcal{C}_{\max }(n, \varepsilon)$ be the $(n, \varepsilon)$-capacity region in the case that, along with the replacements in the previous step, (13) is replaced by

$$
\max _{m_{1} \in\left[M_{1, n}\right], m_{2} \in\left[M_{2, n}\right]} \operatorname{Pr}\left(\left(\mathrm{M}_{1}, \mathrm{M}_{2}\right) \neq\left(\hat{\mathrm{M}}_{1}, \hat{\mathrm{M}}_{2}\right) \mid\left(\mathrm{M}_{1}, \mathrm{M}_{2}\right)=\left(m_{1}, m_{2}\right)\right) \leq \varepsilon_{n} .
$$

That is, the average error probability is replaced by the maximal error probability. Here we show that $\mathcal{C}(n, \varepsilon)$ and $\mathcal{C}_{\max }(n, \varepsilon)$ are equivalent for the purposes of second-order asymptotics, thus allowing us to focus on the maximal error probability for the converse proof.

\footnotetext{
${ }^{1}$ There may be some imprecision in the use of the word "backoff" here as for angles in the second (resp. fourth) quadrant, $L_{2}$ (resp. $L_{1}$ ) is positive. On the other hand, one could generally refer to "backoff" as moving in some inward direction relative to the capacity region boundary, even if it is in a direction where one of the second-order rates increases. The same goes for the term "addition".
} 
By combining ideas from Csiszár-Körner [24, Lem. 16.2] and Polyanskiy [25, Sec 3.4.4], we will start with the average-error code, and use an expurgation argument to obtain a maximal-error code having the same asymptotic rates and error probability. Let $\varepsilon_{n}\left(m_{1}, m_{2}\right)$ be the error probability given that the message pair $\left(m_{1}, m_{2}\right)$ is encoded, and let

$$
\varepsilon_{n}\left(m_{2}\right):=\frac{1}{M_{1, n}} \sum_{m_{1}=1}^{M_{1, n}} \varepsilon_{n}\left(m_{1}, m_{2}\right)
$$

be the error probability for message $m_{2}$, averaged over $\mathrm{M}_{1}$.

Consider a sequence of codes with message sets $\mathcal{M}_{1, n}$ and $\mathcal{M}_{2, n}$, having an error probability not exceeding $\varepsilon_{n}$. Let $\tilde{\mathcal{M}}_{2, n}$ contain the fraction $\frac{1}{\sqrt{n}}$ of the messages $m_{2} \in \mathcal{M}_{2, n}$ with the highest values of $\varepsilon_{n}\left(m_{2}\right)$ (here and subsequently, we ignore rounding issues, since these do not affect the argument). It follows that

$$
\varepsilon_{n}\left(m_{2}\right) \leq \frac{\varepsilon_{n}}{1-\frac{1}{\sqrt{n}}}
$$

since otherwise the codewords not appearing in $\tilde{\mathcal{M}}_{2, n}$ would contribute more than $\varepsilon_{n}$ to the average error probability of the original code, causing a contradiction.

Before proceeding, we observe the simple fact that for each $m_{2}$, we can arbitrarily re-arrange the codewords $\left\{\mathbf{x}_{1}\left(m_{1}, m_{2}\right)\right\}_{m_{1}=1}^{M_{1, n}}$ (e.g. interchanging the codewords corresponding to two different $m_{1}$ values) without changing the average or maximal error probability. In contrast, for the standard MAC, $\mathbf{x}_{1}$ can only depend on $m_{1}$, meaning that such a re-arrangement cannot be done separately for each value of $m_{2}$. Thus, the assumption of degraded message sets is crucial in the following arguments. This should be unsurprising, since the capacity regions for the average and maximal error differ in general for the standard MAC [26].

For each $m_{2} \in \tilde{\mathcal{M}}_{2, n}$, let $\tilde{\mathcal{M}}_{1, n}\left(m_{2}\right)$ contain the fraction $\frac{1}{\sqrt{n}}$ of the messages $m_{1}$ with the highest values of $\varepsilon_{n}\left(m_{1}, m_{2}\right)$. By relabeling the codewords in accordance with the previous paragraph if necessary, we can assume that $\tilde{\mathcal{M}}_{1, n}:=\tilde{\mathcal{M}}_{1, n}\left(m_{2}\right)$ is the same for each $m_{2}$. Repeating the argument following (51), we conclude that

$$
\varepsilon_{n}\left(m_{1}, m_{2}\right) \leq \frac{\varepsilon_{n}\left(m_{2}\right)}{1-\frac{1}{\sqrt{n}}} \leq \frac{\varepsilon_{n}}{\left(1-\frac{1}{\sqrt{n}}\right)^{2}}=\varepsilon_{n}+O\left(\frac{1}{\sqrt{n}}\right)
$$

for all $m_{1} \in \tilde{\mathcal{M}}_{1, n}$ and $m_{2} \in \tilde{\mathcal{M}}_{2, n}$. Moreover, we have by construction that

$$
\frac{1}{n} \log \left|\tilde{\mathcal{M}}_{j, n}\right|=\frac{1}{n} \log \left|\mathcal{M}_{j, n}\right|-\frac{\log n}{2 n}
$$

for $j=1,2$. By absorbing the remainder terms in (53) and (54) into the third-order term $\bar{g}(\rho, \varepsilon, n)$ in (35), we see that it suffices to prove the converse result for the maximal error probability.

3) Correlation Type Classes: Define $\mathcal{I}_{0}:=\{0\}$ and $\mathcal{I}_{k}:=\left(\frac{k-1}{n}, \frac{k}{n}\right], k \in[n]$, and let $\mathcal{I}_{-k}:=-\mathcal{I}_{k}$ for $k \in[n]$. We see that the family $\left\{\mathcal{I}_{k}: k \in[-n: n]\right\}$ forms a partition of $[-1,1]$. Consider the correlation type classes (or simply type classes)

$$
\mathcal{T}_{n}(k):=\left\{\left(\mathbf{x}_{1}, \mathbf{x}_{2}\right): \frac{\left\langle\mathbf{x}_{1}, \mathbf{x}_{2}\right\rangle}{\left\|\mathbf{x}_{1}\right\|_{2}\left\|\mathbf{x}_{2}\right\|_{2}} \in \mathcal{I}_{k}\right\}
$$

where $k \in[-n: n]$, and $\left\langle\mathbf{x}_{1}, \mathbf{x}_{2}\right\rangle:=\sum_{i=1}^{n} x_{1 i} x_{2 i}$ is the standard inner product in $\mathbb{R}^{n}$. The total number of type classes is $2 n+1$, which is polynomial in $n$ analogously to the case of discrete alphabets [24, Ch. 2].

Here we perform a further reduction (along with those in the first two steps) to codes for which all codeword pairs have the same type. Let the codebook $\mathcal{C}:=\left\{\left(\mathbf{x}_{1}\left(m_{1}, m_{2}\right), \mathbf{x}_{2}\left(m_{2}\right)\right): m_{1} \in \mathcal{M}_{1, n}, m_{2} \in \mathcal{M}_{2, n}\right\}$ be given; in accordance with the previous two steps, we assume that it has codewords meeting the power constraints with equality, and maximal error probability not exceeding $\varepsilon_{n}$. For each $m_{2} \in \mathcal{M}_{2, n}$, we can find a set $\tilde{\mathcal{M}}_{1, n}\left(m_{2}\right) \subset \mathcal{M}_{1, n}$ (reusing the notation of the previous step) such that all pairs of codewords $\left(\mathbf{x}_{1}\left(m_{1}, m_{2}\right), \mathbf{x}_{2}\left(m_{2}\right)\right), m_{1} \in \tilde{\mathcal{M}}_{1, n}\left(m_{2}\right)$ have the same type, say indexed by $k\left(m_{2}\right) \in[-n: n]$, and such that

$$
\frac{1}{n} \log \left|\tilde{\mathcal{M}}_{1, n}\left(m_{2}\right)\right| \geq \frac{1}{n} \log \left|\mathcal{M}_{1, n}\left(m_{2}\right)\right|-\frac{\log (2 n+1)}{n}, \quad \forall m_{2} \in \mathcal{M}_{2, n} .
$$

We may assume that all the sets $\tilde{\mathcal{M}}_{1, n}\left(m_{2}\right), m_{2} \in \mathcal{M}_{2, n}$ have the same cardinality; otherwise, we can remove extra codeword pairs from some sets $\tilde{\mathcal{M}}_{1, n}\left(m_{2}\right)$ and (56) will still be satisfied. Similarly to the previous step, we may 
assume (by relabeling if necessary) that $\tilde{\mathcal{M}}_{1, n}:=\tilde{\mathcal{M}}_{1, n}\left(m_{2}\right)$ is the same for each $m_{2}$. We now have a subcodebook $\tilde{\mathcal{C}}_{1}:=\left\{\left(\mathbf{x}_{1}\left(m_{1}, m_{2}\right), \mathbf{x}_{2}\left(m_{2}\right)\right): m_{1} \in \tilde{\mathcal{M}}_{1, n}, m_{2} \in \mathcal{M}_{2, n}\right\}$, where for each $m_{2}$, all the codeword pairs have the same type and (56) is satisfied. Across the $m_{2}$ 's, there may be different types indexed by $k\left(m_{2}\right) \in[-n: n]$, but there exists a dominant type indexed by $k^{*} \in\left\{k\left(m_{2}\right): m_{2} \in \mathcal{M}_{2, n}\right\}$ and a set $\tilde{\mathcal{M}}_{2, n} \subset \mathcal{M}_{2, n}$ such that

$$
\frac{1}{n} \log \left|\tilde{\mathcal{M}}_{2, n}\right| \geq \frac{1}{n} \log \left|\mathcal{M}_{2, n}\right|-\frac{\log (2 n+1)}{n} .
$$

As such, we have shown that there exists a subcodebook $\tilde{\mathcal{C}}_{12}:=\left\{\left(\mathbf{x}_{1}\left(m_{1}, m_{2}\right), \mathbf{x}_{2}\left(m_{2}\right)\right): m_{1} \in \tilde{\mathcal{M}}_{1, n}, m_{2} \in \tilde{\mathcal{M}}_{2, n}\right\}$ of constant type indexed by $k^{*}$ whose sum rate satisfies

$$
\frac{1}{n} \log \left|\tilde{\mathcal{M}}_{1, n} \times \tilde{\mathcal{M}}_{2, n}\right| \geq \frac{1}{n} \log \left|\mathcal{M}_{1, n} \times \mathcal{M}_{2, n}\right|-\frac{2 \log (2 n+1)}{n} .
$$

The reduced code clearly has a maximal error probability no larger than that $\mathcal{C}$. Combining this observation with (57) and (58), we see that the converse part of Theorem 2 for fixed-type codes implies the same for general codes, since the additional $O\left(\frac{\log n}{n}\right)$ factors in (57) and (58) can be absorbed into the third-order term $\bar{g}(\rho, \varepsilon, n)$. Thus, in the remainder of the proof, we limit our attention to fixed-type codes. For each $n$, the type is indexed by $k \in[-n: n]$, and we define $\hat{\rho}:=\frac{k}{n} \in[-1,1]$. In some cases, we will be interested in sequences of such values, in which case we will make the dependence on $n$ explicit by writing $\hat{\rho}_{n}$.

4) A Verdú-Han-type Converse Bound: We now state a non-asymptotic converse bound based on analogous bounds in Han's work on the information spectrum approach for the general MAC [27, Lem. 4] and in BoucheronSalamatian's work on the information spectrum approach for the general broadcast channel with degraded message sets [28, Lem. 2]. The bound only requires that the average error probability is no larger than $\varepsilon_{n}$, which is guaranteed by the fact that the maximal error probability is no larger than $\varepsilon_{n}$. That is, the reduction to the maximal error probability in Section VI-A2 was performed for the sole purpose of making the reduction to fixed types in Section VI-A3 possible.

Proposition 4. Fix a blocklength $n \geq 1$, auxiliary output distributions $Q_{\mathbf{Y} \mid \mathbf{X}_{2}}$ and $Q_{\mathbf{Y}}$, and a constant $\gamma>0$. For any $\left(n, M_{1}, M_{2}, S_{1}, S_{2}, \varepsilon\right)$-code with codewords of fixed empirical powers $S_{1}$ and $S_{2}$ falling into a single correlation type class $\mathcal{T}_{n}(k)$, there exist random vectors $\left(\mathbf{X}_{1}, \mathbf{X}_{2}\right)$ with joint distribution $P_{\mathbf{X}_{1}, \mathbf{X}_{2}}$ supported on $\left\{\left(\mathbf{x}_{1}, \mathbf{x}_{2}\right) \in \mathcal{T}_{n}(k):\left\|\mathbf{x}_{j}\right\|_{2}^{2}=n S_{j}, j=1,2\right\}$ such that

$$
\varepsilon \geq \operatorname{Pr}(\mathcal{A} \cup \mathcal{B})-2 e^{-n \gamma},
$$

where

$$
\begin{aligned}
\mathcal{A} & :=\left\{\frac{1}{n} \log \frac{W^{n}\left(\mathbf{Y} \mid \mathbf{X}_{1}, \mathbf{X}_{2}\right)}{Q_{\mathbf{Y} \mid \mathbf{X}_{2}}\left(\mathbf{Y} \mid \mathbf{X}_{2}\right)} \leq \frac{1}{n} \log M_{1}-\gamma\right\} \\
\mathcal{B} & :=\left\{\frac{1}{n} \log \frac{W^{n}\left(\mathbf{Y} \mid \mathbf{X}_{1}, \mathbf{X}_{2}\right)}{Q_{\mathbf{Y}}(\mathbf{Y})} \leq \frac{1}{n} \log \left(M_{1} M_{2}\right)-\gamma\right\}
\end{aligned}
$$

with $\mathbf{Y} \mid\left\{\mathbf{X}_{1}=\mathbf{x}_{1}, \mathbf{X}_{2}=\mathbf{x}_{2}\right\} \sim W^{n}\left(\cdot \mid \mathbf{x}_{1}, \mathbf{x}_{2}\right)$.

Proof: The proof is nearly identical to those appearing in [27]-[29], so we omit the details. The starting point is the basic identity

$$
\varepsilon \geq \operatorname{Pr}(\mathcal{A} \cup \mathcal{B})-\operatorname{Pr}(\mathcal{A} \cap \text { no error })-\operatorname{Pr}(\mathcal{B} \cap \text { no error })
$$

We can upper bound the second probability by $e^{-n \gamma}$ by explicitly writing it in terms of the distributions of the codewords and the channel, and using (60) to upper bound $W^{n}$ by $Q_{\mathbf{Y} \mid \mathbf{X}_{2}} M_{1} e^{-n \gamma}$. Handling the third term in (62) similarly yields a second $e^{-n \gamma}$ term, thus resulting in (59).

There are several differences in Proposition 4 compared to [27, Lem. 4]. First, in our work, there are constraints on the codewords, and the support of the input distribution $P_{\mathbf{X}_{1}, \mathbf{X}_{2}}$ is specified to reflect this. Second, there are two (instead of three) events in the probability in (59) because the informed encoder $f_{1, n}$ has access to both messages. Third, we can choose arbitrary output distributions $Q_{\mathbf{Y} \mid \mathbf{X}_{2}}$ and $Q_{\mathbf{Y}}$. This generalization is analogous to the nonasymptotic converse bound by Hayashi and Nagaoka for classical-quantum channels [29, Lem. 4]. The freedom to choose the output distribution is crucial in both our problem and [29]. 
5) Evaluation of the Verdú-Han Bound for $\hat{\rho} \in(-1,1)$ : Recall from Sections VI-A1 and VI-A3 that the codewords satisfy exact power constraints and belong to a single type class $\mathcal{T}_{n}(k)$. In this subsection, we consider the case that $\hat{\rho}:=\frac{k}{n} \in(-1,1)$, and we derive bounds that will be useful for sequences $\hat{\rho}_{n}$ bounded away from -1 and 1. In Section VI-A6, we present alternative bounds to handle the case that $\hat{\rho}_{n} \rightarrow \pm 1$.

We set $\gamma:=\frac{\log n}{2 n}$ in (59), yielding $2 e^{-n \gamma}=\frac{2}{\sqrt{n}}$. Moreover, we choose the output distributions $Q_{\mathbf{Y} \mid \mathbf{X}_{2}}$ and $Q_{\mathbf{Y}}$ to be the $n$-fold products of $Q_{Y \mid X_{2}}$ and $Q_{Y}$, defined in (27)-(28) respectively, with $\hat{\rho}$ in place of $\rho$.

We now characterize the statistics of the first and second moments of $\sum_{i=1}^{n} \mathbf{j}\left(x_{1 i}, x_{2 i}, Y_{i}\right)$ in (29) for fixed sequences $\left(\mathbf{x}_{1}, \mathbf{x}_{2}\right) \in \mathcal{T}_{n}(k)$. From Appendix $\mathrm{A}$, these moments can be expressed as affine functions of the empirical powers $\frac{1}{n}\left\|\mathbf{x}_{1}\right\|_{2}^{2}, \frac{1}{n}\left\|\mathbf{x}_{2}\right\|_{2}^{2}$ and the empirical correlation coefficient $\frac{\left\langle\mathbf{x}_{1}, \mathbf{x}_{2}\right\rangle}{\left\|\mathbf{x}_{1}\right\|_{2}\left\|\mathbf{x}_{2}\right\|_{2}}$. The former two quantities are fixed due to the reduction in Section VI-A1, and the latter is within $\frac{1}{n}$ of $\hat{\rho}$ by the assumption that $\left(\mathbf{x}_{1}, \mathbf{x}_{2}\right) \in \mathcal{T}_{n}(k)$. Moreover, a direct substitution into (A.6) and A.12 reveals that the mean vector and covariance matrix coincide with $\mathbf{I}(\hat{\rho})$ and $\mathbf{V}(\hat{\rho})$ when $\frac{\left\langle\mathbf{x}_{1}, \mathbf{x}_{2}\right\rangle}{\left\|\mathbf{x}_{1}\right\|_{2}\left\|\mathbf{x}_{2}\right\|_{2}}$ is precisely equal to $\hat{\rho}$. Combining the preceding observations, we obtain

$$
\begin{array}{r}
\left\|\mathbb{E}\left[\frac{1}{n} \sum_{i=1}^{n} \mathbf{j}\left(x_{1 i}, x_{2 i}, Y_{i}\right)\right]-\mathbf{I}(\hat{\rho})\right\|_{\infty} \leq \frac{\xi_{1}}{n} \\
\left\|\operatorname{Cov}\left[\frac{1}{\sqrt{n}} \sum_{i=1}^{n} \mathbf{j}\left(x_{1 i}, x_{2 i}, Y_{i}\right)\right]-\mathbf{V}(\hat{\rho})\right\|_{\infty} \leq \frac{\xi_{2}}{n}
\end{array}
$$

for $\mathbf{Y} \sim W^{n}\left(\cdot \mid \mathbf{x}_{1}, \mathbf{x}_{2}\right)$, where $\xi_{1}>0$ and $\xi_{2}>0$ are constants. Moreover, we can take these constants to be independent of $\hat{\rho}$, since the corresponding coefficients in $\mathrm{A} .6$ and $\mathrm{A}$.12 are uniformly bounded.

Let $R_{j, n}:=\frac{1}{n} \log M_{j, n}$ for $j=1,2$, and let $\mathbf{R}_{n}:=\left[R_{1, n}, R_{1, n}+R_{2, n}\right]^{T}$. We have

$$
\operatorname{Pr}(\mathcal{A} \cup \mathcal{B})=1-\operatorname{Pr}\left(\mathcal{A}^{c} \cap \mathcal{B}^{c}\right)=1-\mathbb{E}_{\mathbf{X}_{1}, \mathbf{X}_{2}}\left[\operatorname{Pr}\left(\mathcal{A}^{c} \cap \mathcal{B}^{c} \mid \mathbf{X}_{1}, \mathbf{X}_{2}\right)\right]
$$

and in particular, using the definition of $\mathbf{j}\left(x_{1}, x_{2}, y\right)$ in 29$)$ and the fact that $Q_{\mathbf{Y} \mid \mathbf{X}_{2}}$ and $Q_{\mathbf{Y}}$ are product distributions,

$$
\begin{aligned}
\operatorname{Pr}\left(\mathcal{A}^{c} \cap \mathcal{B}^{c} \mid \mathbf{x}_{1}, \mathbf{x}_{2}\right) & =\operatorname{Pr}\left(\frac{1}{n} \sum_{i=1}^{n} \mathbf{j}\left(x_{1 i}, x_{2 i}, Y_{i}\right)>\mathbf{R}_{n}-\gamma \mathbf{1}\right) \\
& \leq \operatorname{Pr}\left(\frac{1}{n} \sum_{i=1}^{n}\left(\mathbf{j}\left(x_{1 i}, x_{2 i}, Y_{i}\right)-\mathbb{E}\left[\mathbf{j}\left(x_{1 i}, x_{2 i}, Y_{i}\right)\right]\right)>\mathbf{R}_{n}-\mathbf{I}(\hat{\rho})-\gamma \mathbf{1}-\frac{\xi_{1}}{n} \mathbf{1}\right),
\end{aligned}
$$

where (67) follows from (63).

We are now in a position to apply the multivariate Berry-Esseen theorem [30], [31] (see Appendix B). The first two moments are bounded according to (63)-(64), and in Appendix A we show that, upon replacing the given $\left(\mathbf{x}_{1}, \mathbf{x}_{2}\right)$ pair by a different pair yielding the same statistics of $\sum_{i=1}^{n} \mathbf{j}\left(x_{1 i}, x_{2 i}, Y_{i}\right)$ if necessary (cf. Lemma 9), the required third moment is uniformly bounded (cf. Lemma 10). It follows that

$$
\begin{aligned}
& \operatorname{Pr}\left(\mathcal{A}^{c} \cap \mathcal{B}^{c} \mid \mathbf{x}_{1}, \mathbf{x}_{2}\right) \\
& \leq \Psi\left(\sqrt{n}\left(I_{1}(\hat{\rho})+\gamma+\frac{\xi_{1}}{n}-R_{1, n}\right), \sqrt{n}\left(I_{12}(\hat{\rho})+\gamma+\frac{\xi_{1}}{n}-\left(R_{1, n}+R_{2, n}\right)\right) ; \operatorname{Cov}\left[\frac{1}{\sqrt{n}} \sum_{i=1}^{n} \mathbf{j}\left(x_{1 i}, x_{2 i}, Y_{i}\right)\right]\right)+\frac{\psi(\hat{\rho})}{\sqrt{n}},
\end{aligned}
$$

where $\psi(\hat{\rho})$ represents the remainder term. By Taylor expanding the continuously differentiable function $\left(z_{1}, z_{2}, \mathbf{V}\right) \mapsto$ $\Psi\left(z_{1}, z_{2} ; \mathbf{V}\right)$, and using the approximation in (64) and the fact that $\operatorname{det}(\mathbf{V}(\hat{\rho}))>0$ for $\hat{\rho} \in(-1,1)$, we obtain

$$
\operatorname{Pr}\left(\mathcal{A}^{c} \cap \mathcal{B}^{c} \mid \mathbf{x}_{1}, \mathbf{x}_{2}\right) \leq \Psi\left(\sqrt{n}\left(I_{1}(\hat{\rho})-R_{1, n}\right), \sqrt{n}\left(I_{12}(\hat{\rho})-\left(R_{1, n}+R_{2, n}\right)\right) ; \mathbf{V}(\hat{\rho})\right)+\frac{\eta(\hat{\rho}) \log n}{\sqrt{n}}
$$

for some suitable remainder term $\eta(\hat{\rho})$. It should be noted that $\psi(\hat{\rho}), \eta(\hat{\rho}) \rightarrow \infty$ as $\hat{\rho} \rightarrow \pm 1$, since $\mathbf{V}(\hat{\rho})$ becomes singular as $\hat{\rho} \rightarrow \pm 1$. Despite this non-uniformity, we conclude from (59), 65) and (69) that any $(n, \varepsilon)$-code with codewords in $\mathcal{T}_{n}(k)$ must have rates that satisfy

$$
\left[\begin{array}{c}
R_{1, n} \\
R_{1, n}+R_{2, n}
\end{array}\right] \in \mathbf{I}(\hat{\rho})+\frac{\Psi^{-1}\left(\mathbf{V}(\hat{\rho}), \varepsilon+\frac{2}{\sqrt{n}}+\frac{\eta(\hat{\rho}) \log n}{\sqrt{n}}\right)}{\sqrt{n}} .
$$


The following "continuity" lemma for $\varepsilon \mapsto \Psi^{-1}(\mathbf{V}, \varepsilon)$ is proved in Appendix C

Lemma 5. Fix $0<\varepsilon<1$ and a positive sequence $\lambda_{n}=o(1)$. Let $\mathbf{V}$ be a non-zero positive semi-definite matrix. There exists a function $h(\mathbf{V}, \varepsilon)$ such that

$$
\Psi^{-1}\left(\mathbf{V}, \varepsilon+\lambda_{n}\right) \subset \Psi^{-1}(\mathbf{V}, \varepsilon)+h(\mathbf{V}, \varepsilon) \lambda_{n} \mathbf{1},
$$

and such that $h(\mathbf{V}(\rho), \varepsilon)$ is finite for each $\rho \neq \pm 1$, while being possibly divergent only as $\rho \rightarrow \pm 1$.

We conclude from Lemma 5 that

$$
\Psi^{-1}\left(\mathbf{V}(\hat{\rho}), \varepsilon+\frac{2}{\sqrt{n}}+\frac{\eta(\hat{\rho}) \log n}{\sqrt{n}}\right) \subset \Psi^{-1}(\mathbf{V}(\hat{\rho}), \varepsilon)+\frac{h(\hat{\rho}, \varepsilon) \log n}{\sqrt{n}} \mathbf{1}
$$

where $h(\hat{\rho}, \varepsilon):=h(\mathbf{V}(\hat{\rho}), \varepsilon)$ diverges only as $\hat{\rho} \rightarrow \pm 1$. Uniting (70) and (72), we deduce that

$$
\left[\begin{array}{c}
R_{1, n} \\
R_{1, n}+R_{2, n}
\end{array}\right] \in \mathbf{I}(\hat{\rho})+\frac{\Psi^{-1}(\mathbf{V}(\hat{\rho}), \varepsilon)}{\sqrt{n}}+\frac{h(\hat{\rho}, \varepsilon) \log n}{n} \mathbf{1} .
$$

6) Evaluation of the Verdú-Han Bound with $\hat{\rho}_{n} \rightarrow \pm 1$ : Here we consider a sequence of codes of a single type indexed by $k_{n}$ such that $\hat{\rho}_{n}:=\frac{k_{n}}{n} \rightarrow 1$. The case $\hat{\rho}_{n} \rightarrow-1$ is handled similarly, and the details are thus omitted. Our aim is to show that

$$
\left[\begin{array}{c}
R_{1, n} \\
R_{1, n}+R_{2, n}
\end{array}\right] \in \mathbf{I}\left(\hat{\rho}_{n}\right)+\frac{\Psi^{-1}\left(\mathbf{V}\left(\hat{\rho}_{n}\right), \varepsilon\right)}{\sqrt{n}}+o\left(\frac{1}{\sqrt{n}}\right) \mathbf{1} .
$$

The following lemma states that as $\hat{\rho}_{n} \rightarrow 1$, the set $\Psi^{-1}\left(\mathbf{V}\left(\hat{\rho}_{n}\right), \varepsilon\right)$ in (74) can be approximated by $\Psi^{-1}(\mathbf{V}(1), \varepsilon)$, which is a simpler rectangular set. The proof of the lemma is provided in Appendix D

Lemma 6. Fix $0<\varepsilon<1$ and a sequence $\left\{\rho_{n}\right\}$ such that $\hat{\rho}_{n} \rightarrow 1$. There exist positive sequences $a_{n}, b_{n}=$ $\Theta\left(\left(1-\hat{\rho}_{n}\right)^{1 / 4}\right)$ and $c_{n}=\Theta\left(\left(1-\hat{\rho}_{n}\right)^{1 / 2}\right)$ satisfying

$$
\left[\begin{array}{c}
0 \\
\sqrt{V_{12}(1)} \Phi^{-1}\left(\varepsilon+a_{n}\right)
\end{array}\right]^{-}-b_{n} \mathbf{1} \subset \Psi^{-1}\left(\mathbf{V}\left(\hat{\rho}_{n}\right), \varepsilon\right) \subset\left[\sqrt{V_{12}(1)} \Phi^{-1}(\varepsilon)\right]^{-}+c_{n} \mathbf{1}
$$

From the inner bound in Lemma 6, in order to show (74) it suffices to show

$$
\left[\begin{array}{c}
R_{1, n} \\
R_{1, n}+R_{2, n}
\end{array}\right] \leq \mathbf{I}\left(\hat{\rho}_{n}\right)+\sqrt{\frac{V_{12}(1)}{n}}\left[\begin{array}{c}
0 \\
\Phi^{-1}(\varepsilon)
\end{array}\right]+o\left(\frac{1}{\sqrt{n}}\right) \mathbf{1}
$$

where we absorbed the sequences $a_{n}, b_{n}$ into the $o\left(\frac{1}{\sqrt{n}}\right)$ term.

We return to the step in 67), which when combined with the Verdú-Han-type bound in Proposition 4 (with $\left.\gamma:=\frac{\log n}{2 n}\right)$ yields for some $\left(\mathbf{x}_{1}, \mathbf{x}_{2}\right) \in \mathcal{T}_{n}(k)$ that

$$
\begin{aligned}
\varepsilon_{n} \geq 1 & -\operatorname{Pr}\left(\frac{1}{n} \sum_{i=1}^{n}\left(\mathbf{j}\left(x_{1 i}, x_{2 i}, Y_{i}\right)-\mathbb{E}\left[\mathbf{j}\left(x_{1 i}, x_{2 i}, Y_{i}\right)\right]\right)>\mathbf{R}_{n}-\mathbf{I}\left(\hat{\rho}_{n}\right)-\gamma \mathbf{1}-\frac{\xi_{1}}{n} \mathbf{1}\right)-\frac{2}{\sqrt{n}} \\
\geq \max & \left\{\operatorname{Pr}\left(\frac{1}{n} \sum_{i=1}^{n}\left(j_{1}\left(x_{1 i}, x_{2 i}, Y_{i}\right)-\mathbb{E}\left[j_{1}\left(x_{1 i}, x_{2 i}, Y_{i}\right)\right]\right) \leq R_{1, n}-I_{1}\left(\hat{\rho}_{n}\right)-\gamma-\frac{\xi_{1}}{n}\right),\right. \\
& \left.\quad \operatorname{Pr}\left(\frac{1}{n} \sum_{i=1}^{n}\left(j_{12}\left(x_{1 i}, x_{2 i}, Y_{i}\right)-\mathbb{E}\left[j_{12}\left(x_{1 i}, x_{2 i}, Y_{i}\right)\right]\right) \leq R_{1, n}+R_{2, n}-I_{12}\left(\hat{\rho}_{n}\right)-\gamma-\frac{\xi_{1}}{n}\right)\right\}-\frac{2}{\sqrt{n}} .
\end{aligned}
$$

From (64) and the assumption that $\hat{\rho}_{n} \rightarrow 1$, the variance of $\sum_{i=1}^{n} j_{12}\left(x_{1 i}, x_{2 i}, Y_{i}\right)$ equals $n\left(V_{12}(1)+o(1)\right)$. Since $V_{12}(1)>0$, we can treat the second term in the maximum in (78) in an identical fashion to the single-user setting [7], [8] to obtain the second of the element-wise inequalities in (76). It remains to prove the first, i.e. to show that no $\Theta\left(\frac{1}{\sqrt{n}}\right)$ addition to $R_{1, n}$ is possible for $\varepsilon \in(0,1)$.

Since $V_{1}(1)=1$ and $V_{1}(\cdot)$ is continuous in $\rho$, we have $V_{1}\left(\hat{\rho}_{n}\right) \rightarrow 0$. Combining this observation with (64), we conclude that the variance of $\sum_{i=1}^{n} j_{1}\left(x_{1 i}, x_{2 i}, Y_{i}\right)$ is $o(n)$, and we thus have from Chebyshev's inequality that

$$
\operatorname{Pr}\left(\frac{1}{n} \sum_{i=1}^{n}\left(j_{1}\left(x_{1 i}, x_{2 i}, Y_{i}\right)-\mathbb{E}\left[j_{1}\left(x_{1 i}, x_{2 i}, Y_{i}\right)\right]\right) \leq \frac{c}{\sqrt{n}}\right) \rightarrow 1
$$

for all $c>0$. Substituting (79) into (78) and taking $c \rightarrow 0$ yields $R_{1, n} \leq I_{1}\left(\hat{\rho}_{n}\right)+o\left(\frac{1}{\sqrt{n}}\right)$, as desired. 
7) Completion of the Proof: Combining (73) and (74), we conclude that for any sequence of codes with error probability not exceeding $\varepsilon \in(0,1)$, we have for some sequence $\hat{\rho}_{n} \in[-1,1]$ that

$$
\left[\begin{array}{c}
R_{1, n} \\
R_{1, n}+R_{2, n}
\end{array}\right] \in \mathbf{I}\left(\hat{\rho}_{n}\right)+\frac{\Psi^{-1}\left(\mathbf{V}\left(\hat{\rho}_{n}\right), \varepsilon\right)}{\sqrt{n}}+\bar{g}\left(\hat{\rho}_{n}, \varepsilon, n\right) \mathbf{1}
$$

where $\bar{g}(\rho, \varepsilon, n)$ satisfies the conditions in the theorem statement. Specifically, the first condition follows from (73) (with $\bar{g}(\rho, \varepsilon, n):=h(\rho, \varepsilon) \frac{\log n}{n}$ ), and the second from (74) (with $\bar{g}(\rho, \varepsilon, n)=o\left(\frac{1}{\sqrt{n}}\right)$ ). This concludes the proof of the global converse.

\section{B. Direct Part}

We now prove the inner bound in (36). At a high level, we will adopt the strategy of drawing random codewords on appropriate spheres, similarly to Polyanskiy et al. [8, Thm. 54] and Tan-Tomamichel [9].

1) Random-Coding Ensemble: Let $\rho \in[0,1]$ be a fixed correlation parameter. The ensemble will be defined in such a way that, with probability one, each codeword pair falls into the set

$$
\mathcal{D}_{n}(\rho):=\left\{\left(\mathbf{x}_{1}, \mathbf{x}_{2}\right):\left\|\mathbf{x}_{1}\right\|_{2}^{2}=n S_{1},\left\|\mathbf{x}_{2}\right\|_{2}^{2}=n S_{2},\left\langle\mathbf{x}_{1}, \mathbf{x}_{2}\right\rangle=n \rho \sqrt{S_{1} S_{2}}\right\} .
$$

This means that the power constraints in (10) are satisfied with equality, and the empirical correlation between each codeword pair is exactly $\rho$. We use superposition coding, in which the codewords are generated according to

$$
\left\{\left(\mathbf{X}_{2}\left(m_{2}\right),\left\{\mathbf{X}_{1}\left(m_{1}, m_{2}\right)\right\}_{m_{1}=1}^{M_{1, n}}\right)\right\}_{m_{2}=1}^{M_{2, n}} \sim \prod_{m_{2}=1}^{M_{2, n}}\left(P_{\mathbf{X}_{2}}\left(\mathbf{x}_{2}\left(m_{2}\right)\right) \prod_{m_{1}=1}^{M_{1, n}} P_{\mathbf{X}_{1} \mid \mathbf{X}_{2}}\left(\mathbf{x}_{1}\left(m_{1}, m_{2}\right) \mid \mathbf{x}_{2}\left(m_{2}\right)\right)\right)
$$

for codeword distributions $P_{\mathbf{X}_{2}}$ and $P_{\mathbf{X}_{1} \mid \mathbf{X}_{2}}$. We choose the codeword distributions to be

$$
\begin{aligned}
P_{\mathbf{X}_{2}}\left(\mathbf{x}_{2}\right) & \propto \delta\left\{\left\|\mathbf{x}_{2}\right\|_{2}^{2}=n S_{2}\right\}, \quad \text { and } \\
P_{\mathbf{X}_{1} \mid \mathbf{X}_{2}}\left(\mathbf{x}_{1} \mid \mathbf{x}_{2}\right) & \propto \delta\left\{\left\|\mathbf{x}_{1}\right\|_{2}^{2}=n S_{1},\left\langle\mathbf{x}_{1}, \mathbf{x}_{2}\right\rangle=n \rho \sqrt{S_{1} S_{2}}\right\},
\end{aligned}
$$

where $\delta\{\cdot\}$ is the Dirac $\delta$-function, and $P_{\mathbf{X}}(\mathbf{x}) \propto \delta\{\mathbf{x} \in \mathcal{A}\}$ means that $P_{\mathbf{X}}(\mathbf{x})=\frac{\delta\{\mathbf{x} \in \mathcal{A}\}}{c}$, with the normalization constant $c>0$ chosen such that $\int_{\mathcal{A}} P_{\mathbf{X}}(\mathbf{x}) \mathrm{d} \mathbf{x}=1$. In other words, each $\mathbf{X}_{2}\left(m_{2}\right), m_{2} \in\left[M_{2, n}\right]$ is drawn uniformly from an $(n-1)$-sphere (i.e. an $(n-1)$-dimensional manifold in $\mathbb{R}^{n}$ ) with radius $\sqrt{n S_{2}}$ and for each $m_{2}$, each $\mathbf{x}_{1}\left(m_{1}, m_{2}\right), m_{1} \in\left[M_{1, n}\right]$ is drawn uniformly from the set of all $\mathbf{x}_{1}$ satisfying the power and correlation coefficient constraints with equality. We will see that this set is in fact an $(n-2)$-sphere of radius $\sqrt{n S_{1}\left(1-\rho^{2}\right)}$, and is thus non-empty for all $\rho \in[0,1]$. These distributions clearly ensure that the codeword pairs belong to $\mathcal{D}_{n}(\rho)$ with probability one.

2) A Feinstein-type Achievability Bound: We now state a non-asymptotic achievability based on an analogous bound for the MAC [27, Lem. 3]. This bound can be considered as a dual of Proposition 4 Define

$$
\begin{aligned}
P_{\mathbf{X}_{1} \mid \mathbf{X}_{2}} W^{n}\left(\mathbf{y} \mid \mathbf{x}_{2}\right) & :=\int_{\mathbb{R}^{n}} P_{\mathbf{X}_{1} \mid \mathbf{X}_{2}}\left(\mathbf{x}_{1} \mid \mathbf{x}_{2}\right) W^{n}\left(\mathbf{y} \mid \mathbf{x}_{1}, \mathbf{x}_{2}\right) \mathrm{d} \mathbf{x}_{1}, \\
P_{\mathbf{X}_{1}, \mathbf{X}_{2}} W^{n}(\mathbf{y}) & :=\int_{\mathbb{R}^{n}} \int_{\mathbb{R}^{n}} P_{\mathbf{X}_{1}, \mathbf{X}_{2}}\left(\mathbf{x}_{1}, \mathbf{x}_{2}\right) W^{n}\left(\mathbf{y} \mid \mathbf{x}_{1}, \mathbf{x}_{2}\right) \mathrm{d} \mathbf{x}_{1} \mathrm{~d} \mathbf{x}_{2}
\end{aligned}
$$

to be output distributions induced by a joint distribution $P_{\mathbf{X}_{1}, \mathbf{X}_{2}}$ and the channel $W^{n}$. Moreover, let $\frac{\mathrm{d} P_{1}}{\mathrm{~d} P_{2}}$ denote the Radom-Nikodym derivative between two probability distributions $P_{1}$ and $P_{2}$.

Proposition 7. Fix a blocklength $n \geq 1$, a joint distribution $P_{\mathbf{X}_{1}, \mathbf{X}_{2}}$ such that $\left\|\mathbf{X}_{1}\right\|_{2}^{2} \leq n S_{1}$ and $\left\|\mathbf{X}_{2}\right\|_{2}^{2} \leq n S_{2}$ almost surely, auxiliary output distributions $Q_{\mathbf{Y} \mid \mathbf{X}_{2}}$ and $Q_{\mathbf{Y}}$, a constant $\gamma>0$, and two sets $\mathcal{A}_{1} \subseteq \mathcal{X}_{2}^{n} \times \mathcal{Y}^{n}$ and $\mathcal{A}_{12} \subseteq \mathcal{Y}^{n}$. Then there exists an $\left(n, M_{1}, M_{2}, S_{1}, S_{2}, \varepsilon\right)$-code for which

$$
\varepsilon \leq \operatorname{Pr}(\mathcal{F} \cup \mathcal{G})+\Lambda_{1} e^{-n \gamma}+\Lambda_{12} e^{-n \gamma}+\operatorname{Pr}\left(\left(\mathbf{X}_{2}, \mathbf{Y}\right) \notin \mathcal{A}_{1}\right)+\operatorname{Pr}\left(\mathbf{Y} \notin \mathcal{A}_{12}\right),
$$

where

$$
\Lambda_{1}:=\sup _{\left(\mathbf{x}_{2}, \mathbf{y}\right) \in \mathcal{A}_{1}} \frac{\mathrm{d} P_{\mathbf{X}_{1} \mid \mathbf{X}_{2}} W^{n}\left(\cdot \mid \mathbf{x}_{2}\right)}{\mathrm{d} Q_{\mathbf{Y} \mid \mathbf{X}_{2}}\left(\cdot \mid \mathbf{x}_{2}\right)}(\mathbf{y}), \quad \Lambda_{12}:=\sup _{\mathbf{y} \in \mathcal{A}_{12}} \frac{\mathrm{d} P_{\mathbf{X}_{1}, \mathbf{X}_{2}} W^{n}}{\mathrm{~d} Q \mathbf{Y}}(\mathbf{y})
$$


and

$$
\begin{aligned}
\mathcal{F} & :=\left\{\frac{1}{n} \log \frac{W^{n}\left(\mathbf{Y} \mid \mathbf{X}_{1}, \mathbf{X}_{2}\right)}{Q_{\mathbf{Y} \mid \mathbf{X}_{2}}\left(\mathbf{Y} \mid \mathbf{X}_{2}\right)} \leq \frac{1}{n} \log M_{1}+\gamma\right\} \\
\mathcal{G} & :=\left\{\frac{1}{n} \log \frac{W^{n}\left(\mathbf{Y} \mid \mathbf{X}_{1}, \mathbf{X}_{2}\right)}{Q_{\mathbf{Y}}(\mathbf{Y})} \leq \frac{1}{n} \log \left(M_{1} M_{2}\right)+\gamma\right\}
\end{aligned}
$$

with $\mathbf{Y} \mid\left\{\mathbf{X}_{1}=\mathbf{x}_{1}, \mathbf{X}_{2}=\mathbf{x}_{2}\right\} \sim W^{n}\left(\cdot \mid \mathbf{x}_{1}, \mathbf{x}_{2}\right)$.

Proof: The proof is essentially identical to [27, Lem. 3] (among others), so we omit the details. We consider superposition coding of the form given in (82), along with a threshold decoder that searches for a codeword pair $\left(x_{1}, x_{2}\right)$ violating the inequalities in (89)-(90). The first term in (87) is the probability that the transmitted pair fails to meet this condition. The two subsequent terms correspond to the probability that some incorrect pair does meet this condition, and are obtained using the union bound and a standard change of measure argument (e.g. see [4]). The final two terms are obtained by treating the events therein as errors (i.e. atypical events), thus permitting the restrictions to $\mathcal{A}_{1}$ and $\mathcal{A}_{12}$ in (88).

The main difference between (87) and traditional Feinstein-type threshold decoding bounds (e.g. [27, Lem. 3], [32. Lem. 1]) is that we have the freedom to choose arbitrary output distributions $Q_{\mathbf{Y} \mid \mathbf{X}_{2}}$ and $Q_{\mathbf{Y}}$; this comes at the cost of introducing the multiplicative factors $\Lambda_{1}$ and $\Lambda_{12}$ that depend on the maximum value of the Radon-Nikodym derivatives in (88). Our bound in (87) allows us to exclude "atypical" values of $\left(\mathbf{x}_{2}, \mathbf{y}\right) \notin \mathcal{A}_{1}$ and $\mathbf{y} \notin \mathcal{A}_{12}$, thus facilitating the bounding of $\Lambda_{1}$ and $\Lambda_{12}$.

As with all analyses involving uniform coding on spheres [8], [9], [15], it is imperative to control $\Lambda_{1}$ and $\Lambda_{12}$. For this purpose, we leverage the following lemma, which is proved in Appendix E For concreteness, we make the dependence of certain quantities appearing in Proposition 7 on $n$ and $\rho$ explicit, e.g. $\Lambda_{1}(n, \rho)$.

Lemma 8. Consider the setup of Proposition 7 where the output distributions are given by $Q_{\mathbf{Y} \mid \mathbf{X}_{2}}:=\left(P_{X_{1} \mid X_{2}} W\right)^{n}$ and $Q_{\mathbf{Y}}:=\left(P_{X_{1}, X_{2}} W\right)^{n}$ with $P_{X_{1}, X_{2}}:=\mathcal{N}(\mathbf{0}, \mathbf{\Sigma}(\rho))$ (see (22) ), and the joint distribution $P_{\mathbf{X}_{1}, \mathbf{X}_{2}}$ is described by (83)-(84). There exist sets $\mathcal{A}_{1}$ and $\mathcal{A}_{12}$ (depending on $n$ and $\rho$ ) such that

$$
\begin{gathered}
\max _{\rho \in[0,1]} \max \left\{\Lambda_{1}(n, \rho), \Lambda_{12}(n, \rho)\right\} \leq \Lambda \\
\max _{\rho \in[0,1]} \max \left\{\operatorname{Pr}\left(\left(\mathbf{X}_{2}, \mathbf{Y}\right) \notin \mathcal{A}_{1}(n, \rho)\right), \operatorname{Pr}\left(\mathbf{Y} \notin \mathcal{A}_{12}(n, \rho)\right)\right\} \leq e^{-n \psi},
\end{gathered}
$$

for all $n>N$, where $\Lambda<\infty, \psi>0$ and $N \in \mathbb{N}$ are constants not depending on $\rho$.

Note that the uniformity of (91)-(92) in $\rho$ is crucial for handling $\rho$ varying with $n$, as is required in Theorem 2

3) Analysis of the Random-Coding Error Probability for $\rho_{n} \rightarrow \rho \in[0,1)$ : We now use Proposition 7 with the joint distribution $P_{\mathbf{X}_{1}, \mathbf{X}_{2}}$ in (83)-(84). By construction, the probability of either codeword violating the power constraint is zero. We choose the output distributions $Q_{\mathbf{Y} \mid \mathbf{X}_{2}}:=\left(P_{X_{1} \mid X_{2}} W\right)^{n}$ and $Q_{\mathbf{Y}}:=\left(P_{X_{1}, X_{2}} W\right)^{n}$ to be of the convenient product form. By using Lemma 8 and Proposition 7 , we obtain

$$
\varepsilon_{n} \leq 1-\operatorname{Pr}\left(\frac{1}{n} \sum_{i=1}^{n} \mathbf{j}\left(X_{1 i}, X_{2 i}, Y_{i}\right)>\mathbf{R}_{n}+\gamma \mathbf{1}\right)+2 \Lambda e^{-n \gamma}+2 e^{-n \psi}
$$

where the information density vector $\mathbf{j}\left(x_{1}, x_{2}, y\right)$ is defined with respect to $P_{X_{1} \mid X_{2}} W\left(y \mid x_{2}\right)$ and $P_{X_{1}, X_{2}} W(y)$, which coincide with $Q_{Y \mid X_{2}}$ and $Q_{Y}$ in (27)-(28). Choosing $\gamma:=\frac{\log n}{2 n}$, we notice that the final term in (93) is $2 \Lambda / \sqrt{n}$. We thus obtain

$$
\varepsilon_{n} \leq \max _{\left(\mathbf{x}_{1}, \mathbf{x}_{2}\right) \in \mathcal{D}_{n}(\rho)} 1-\operatorname{Pr}\left(\frac{1}{n} \sum_{i=1}^{n} \mathbf{j}\left(x_{1 i}, x_{2 i}, Y_{i}\right)>\mathbf{R}_{n}+\gamma \mathbf{1}\right)+\frac{2 \Lambda}{\sqrt{n}}+2 e^{-n \psi} .
$$

Using the definition of $\mathcal{D}_{n}(\rho)$ in (81) and the expressions for the information densities in Appendix A we see that the empirical mean and empirical covariance of the information densities are exactly equal to the true mutual 
information vector and dispersion matrix respectively, i.e.

$$
\begin{aligned}
\mathbb{E}\left[\frac{1}{n} \sum_{i=1}^{n} \mathbf{j}\left(x_{1 i}, x_{2 i}, Y_{i}\right)\right] & =\mathbf{I}(\rho), \quad \text { and } \\
\operatorname{Cov}\left[\frac{1}{\sqrt{n}} \sum_{i=1}^{n} \mathbf{j}\left(x_{1 i}, x_{2 i}, Y_{i}\right)\right] & =\mathbf{V}(\rho)
\end{aligned}
$$

for all $\left(\mathbf{x}_{1}, \mathbf{x}_{2}\right) \in \mathcal{D}_{n}(\rho)$. These are the analogues of (63)- (64) in the converse proof, with the slack parameters $\xi_{1}$ and $\xi_{2}$ replaced by zero. By applying the multivariate Berry-Esseen theorem [30], [31] (see Appendix B) to (94) and performing Taylor expansions similarly to Section VI-A5, we obtain

$$
\varepsilon_{n} \leq 1-\Psi\left(\sqrt{n}\left(I_{1}(\rho)-R_{1, n}\right), \sqrt{n}\left(I_{12}(\rho)-\left(R_{1, n}+R_{2, n}\right)\right) ; \mathbf{V}(\rho)\right)+\frac{\zeta(\rho, \delta) \log n}{\sqrt{n}},
$$

where $\zeta(\rho, \delta)$ is a function depending only on $\rho$ and $\delta$, and diverging only as $\rho \rightarrow 1$. By inverting the relationship between the rates and the error probability similarly to Section VI-A5, we obtain the desired result for any sequence $\left\{\rho_{n}\right\}$ converging to some $\rho \in[0,1)$, i.e. the first part of the theorem.

4) Analysis of the Random-Coding Error Probability for $\rho_{n} \rightarrow 1$ : We now consider a sequence of parameters such that $\rho_{n} \rightarrow 1$. Similarly to (76), it suffices to show the achievability of $\left(R_{1, n}, R_{2, n}\right)$ satisfying

$$
\left[\begin{array}{c}
R_{1, n} \\
R_{1, n}+R_{2, n}
\end{array}\right] \geq \mathbf{I}\left(\rho_{n}\right)+\sqrt{\frac{V_{12}(1)}{n}}\left[\begin{array}{c}
0 \\
\Phi^{-1}(\varepsilon)
\end{array}\right]+o\left(\frac{1}{\sqrt{n}}\right) \mathbf{1}
$$

rather than the equivalent form given by $(80)$; see the outer bound in Lemma 6 ,

Applying the union bound to one minus the probability in (94), we obtain

$$
\varepsilon_{n} \leq \operatorname{Pr}\left(\frac{1}{n} \sum_{i=1}^{n} j_{1}\left(x_{1 i}, x_{2 i}, Y_{i}\right) \leq R_{1, n}+\gamma\right)+\operatorname{Pr}\left(\frac{1}{n} \sum_{i=1}^{n} j_{1}\left(x_{1 i}, x_{2 i}, Y_{i}\right) \leq R_{1, n}+R_{2, n}+\gamma\right)+\frac{2 \Lambda}{\sqrt{n}}+2 e^{-n \psi}
$$

for some $\left(\mathbf{x}_{1}, \mathbf{x}_{2}\right) \in \mathcal{D}_{n}\left(\rho_{n}\right)$. The remaining arguments are again similar to Section VI-A6, so we only provide a brief outline. We fix a small $c>0$ and choose

$$
R_{1, n}=I_{1}\left(\rho_{n}\right)-\frac{c}{\sqrt{n}}-\gamma
$$

Using (95)-96) and applying Chebyshev's inequality similarly to (79), we see that

$$
\operatorname{Pr}\left(\frac{1}{n} \sum_{i=1}^{n} j_{1}\left(x_{1 i}, x_{2 i}, Y_{i}\right) \leq R_{1, n}+\gamma\right) \rightarrow 0
$$

for any $c>0$ (recall that $1-\rho_{n} \rightarrow 0$ implies $V_{1}\left(\rho_{n}\right) \rightarrow 0$ ). Hence, and applying the univariate Berry-Esseen theorem [33, Sec. XVI.5] to the second probability in (99), we obtain (98) and the second part of Theorem 2 .

\section{Proof of Theorem 3; Local Second-Order Result}

\section{A. Converse Part}

We now present the proof of the converse part of Theorem 3 ,

1) Proof for case (i) $(\rho=0)$ : To prove the converse part for case (i), it suffices to consider the most optimistic case, namely $M_{2, n}=1$ (i.e. no information is sent by the uninformed user). From the single-user dispersion result given in [4], [8] (cf. (4)), the number of messages for user 1 must satisfy

$$
\log M_{1, n} \leq n I_{1}(0)+\sqrt{n V_{1}(0)} \Phi^{-1}(\varepsilon)+o(\sqrt{n}),
$$

thus proving the converse part of (43). 
2) Passage to a Convergent Subsequence: In the remainder of the proof, we consider cases (ii) and (iii). Fix a correlation coefficient $\rho \in(0,1]$, and consider any sequence of $\left(n, M_{1, n}, M_{2, n}, S_{1}, S_{2}, \varepsilon_{n}\right)$-codes satisfying (17). Let us consider the associated rates $\left\{\left(R_{1, n}, R_{2, n}\right)\right\}_{n \in \mathbb{N}}$, where $R_{j, n}=\frac{1}{n} \log M_{j, n}$ for $j=1,2$. As required by Definition 4 we suppose that these codes satisfy

$$
\begin{aligned}
\liminf _{n \rightarrow \infty} R_{j, n} & \geq R_{j}^{*}, \\
\liminf _{n \rightarrow \infty} \sqrt{n}\left(R_{j, n}-R_{j}^{*}\right) & \geq L_{j}, \quad j=1,2, \\
\limsup _{n \rightarrow \infty} \varepsilon_{n} & \leq \varepsilon
\end{aligned}
$$

for some $\left(R_{1}^{*}, R_{2}^{*}\right)$ on the boundary parametrized by $\rho$, i.e. $R_{1}^{*}=I_{1}(\rho)$ and $R_{1}^{*}+R_{2}^{*}=I_{12}(\rho)$. The first-order optimality condition in (103) is not explicitly required by Definition 4 but it is implied by (104). Letting $\mathbf{R}_{n}:=$ $\left[R_{1, n}, R_{1, n}+R_{2, n}\right]^{T}$, we have from the global converse bound in (36) that there exists at a (possibly non-unique) sequence $\left\{\rho_{n}\right\}_{n \in \mathbb{N}} \subset[-1,1]$ such that

$$
\mathbf{R}_{n} \in \mathbf{I}\left(\rho_{n}\right)+\frac{\Psi^{-1}\left(\mathbf{V}\left(\rho_{n}\right), \varepsilon\right)}{\sqrt{n}}+\bar{g}\left(\rho_{n}, \varepsilon, n\right) \mathbf{1}
$$

Since we used the liminf for the rates and limsup for the error probability in Definition 4, we may pass to a convergent (but otherwise arbitrary) subsequence of $\left\{\rho_{n}\right\}$, say indexed by $\left\{n_{k}\right\}_{k \in \mathbb{N}}$. Recalling that the liminf (resp. lim sup) is the infimum (resp. supremum) of all subsequential limits, any converse result associated with this subsequence also applies to the original sequence. Note that at least one convergent subsequence is guaranteed to exist, since $[-1,1]$ is compact.

For the sake of clarity, we avoid explicitly writing the subscript $k$. However, it should be understood that asymptotic notations such as $O(\cdot)$ and $(\cdot)_{n} \rightarrow(\cdot)$ are taken with respect to the convergent subsequence.

3) Establishing The Convergence of $\rho_{n}$ to $\rho$ : Although $\bar{g}\left(\rho_{n}, \varepsilon, n\right)$ depends on $\rho_{n}$, we know from Theorem 2 that it is $o\left(\frac{1}{\sqrt{n}}\right)$ for both $\rho_{n} \rightarrow \pm 1$ and $\rho_{n} \rightarrow \rho \in(-1,1)$. Hence, and making use of the previous step, we have

$$
\mathbf{R}_{n} \in \mathbf{I}\left(\rho_{n}\right)+\frac{\Psi^{-1}\left(\mathbf{V}\left(\rho_{n}\right), \varepsilon\right)}{\sqrt{n}}+o\left(\frac{1}{\sqrt{n}}\right) \mathbf{1} .
$$

We claim that this result implies that $\rho_{n}$ converges to $\rho$. Indeed, since the boundary of the capacity region is curved and uniquely parametrized by $\rho$ for $\rho \in(0,1], \rho_{n} \not \rightarrow \rho$ implies for some $\delta>0$ and for all sufficiently large $n$ that either $I_{1}\left(\rho_{n}\right) \leq I_{1}(\rho)-\delta$ or $I_{12}\left(\rho_{n}\right) \leq I_{12}(\rho)-\delta$. We also have from (107) that $R_{1, n} \leq I_{1}\left(\rho_{n}\right)+\frac{\delta}{2}$ and $R_{1, n}+R_{2, n} \leq I_{12}\left(\rho_{n}\right)+\frac{\delta}{2}$ for sufficiently large $n$. Combining these observations, we see that $R_{1, n} \leq I_{1}(\rho)-\frac{\delta}{2}$ or $R_{1, n}+R_{2, n} \leq I_{12}(\rho)-\frac{\delta}{2}$. This, in turn, contradicts the first-order optimality conditions in (103).

4) Taylor Expansion of the Mutual Information Vector: Because each entry of $\mathbf{I}(\rho)$ is twice continuously differentiable, a Taylor expansion yields

$$
\mathbf{I}\left(\rho_{n}\right)=\mathbf{I}(\rho)+\mathbf{D}(\rho)\left(\rho_{n}-\rho\right)+O\left(\left(\rho_{n}-\rho\right)^{2}\right) \mathbf{1},
$$

where $\mathbf{D}(\rho)$ is the derivative of $\mathbf{I}$ defined in (39). In the same way, since each entry of $\mathbf{V}(\rho)$ is continuously differentiable in $\rho$, we have

$$
\left\|\mathbf{V}\left(\rho_{n}\right)-\mathbf{V}(\rho)\right\|_{\infty}=O\left(\rho_{n}-\rho\right) .
$$

We claim that these expansions, along with (107), imply that

$$
\mathbf{R}_{n} \in \mathbf{I}(\rho)+\mathbf{D}(\rho)\left(\rho_{n}-\rho\right)+\frac{\Psi^{-1}(\mathbf{V}(\rho), \varepsilon)}{\sqrt{n}}+\left[o\left(\frac{1}{\sqrt{n}}\right)+O\left(\left(\rho_{n}-\rho\right)^{2}\right)+O\left(\frac{\left(\rho_{n}-\rho\right)^{1 / 2}}{\sqrt{n}}\right)\right] \mathbf{1} .
$$

The final term in the square parentheses results from the outer bound in Lemma 6 for the case $\rho=1$. For $\rho \in(0,1)$ a standard Taylor expansion yields (110) with the last term replaced by $O\left(\frac{\rho_{n}-\rho}{\sqrt{n}}\right)$, and it follows that $(110)$ holds for any given $\rho \in(0,1]$. 
5) Completion of the Proof for Case (ii) $(\rho \in(0,1))$ : Suppose for the time being that $\rho_{n}-\rho=O\left(\frac{1}{\sqrt{n}}\right)$, and hence $\tau_{n}:=\sqrt{n}\left(\rho_{n}-\rho\right)$ is a bounded sequence. By the Bolzano-Weierstrass theorem [34, Thm. 3.6(b)], $\left\{\tau_{n}\right\}$ contains a convergent subsequence, say indexed by $\left\{n_{k}^{\prime}\right\}$; let the limit of this subsequence be $\beta \in \mathbb{R}$. For the blocklengths indexed by $n_{k}^{\prime}$, we know from (110) that

$$
\sqrt{n_{k}^{\prime}}\left(\mathbf{R}_{n_{k}^{\prime}}-\mathbf{I}(\rho)\right) \in \beta \mathbf{D}(\rho)+\Psi^{-1}(\mathbf{V}(\rho), \varepsilon)+o(1) \mathbf{1},
$$

where the $o(1)$ term combines the $o\left(\frac{1}{\sqrt{n}}\right)$ term in (110) and the deviation $\left(\tau_{n_{k}^{\prime}}-\beta\right) \max \left\{-D_{1}(\rho), D_{12}(\rho)\right\}$. From the second-order optimality condition in (104), we know that every convergent subsequence of $\left\{R_{j, n}\right\}_{n \in \mathbb{N}}$ has a subsequential limit that satisfies $\lim _{k \rightarrow \infty} \sqrt{n_{k}}\left(R_{j, n_{k}}-R_{j}^{*}\right) \geq L_{j}$ for $j=1,2$. In other words, for all $\gamma>0$, there exist an integer $K_{1}$ such that

$$
\begin{aligned}
\sqrt{n_{k}^{\prime}}\left(R_{1, n_{k}^{\prime}}-I_{1}(\rho)\right) & \geq L_{1}-\gamma \\
\sqrt{n_{k}^{\prime}}\left(R_{1, n_{k}^{\prime}}+R_{1, n_{k}^{\prime}}-I_{12}(\rho)\right) & \geq L_{1}+L_{2}-2 \gamma
\end{aligned}
$$

for all $k \geq K_{1}$. Thus, we may lower bound the components in the vector on the left of (111) by $L_{1}-\gamma$ and $L_{1}+L_{2}-2 \gamma$. There also exists an integer $K_{2}$ such that the $o(1)$ terms are upper bounded by $\gamma$ for all $k \geq K_{2}$. We conclude that any pair of $\left(\varepsilon, R_{1}^{*}, R_{2}^{*}\right)$-second-order achievable rate pairs $\left(L_{1}, L_{2}\right)$ must satisfy

$$
\left[\begin{array}{c}
L_{1}-2 \gamma \\
L_{1}+L_{2}-3 \gamma
\end{array}\right] \in \bigcup_{\beta \in \mathbb{R}}\left\{\beta \mathbf{D}(\rho)+\Psi^{-1}(\mathbf{V}(\rho), \varepsilon)\right\}
$$

Finally, since $\gamma>0$ is arbitrary, we can take $\gamma \downarrow 0$, thus yielding the right-hand side of (44).

To complete the proof, we must handle the case that $\rho_{n}-\rho$ is not $O\left(\frac{1}{\sqrt{n}}\right)$. By passing to another subsequence if necessary, we may assume that $\rho_{n}-\rho=\omega\left(\frac{1}{\sqrt{n}}\right)$. Roughly speaking, in (110), the term $\frac{1}{\sqrt{n}} \Psi^{-1}(\mathbf{V}(\rho), \varepsilon)$ is dominated by $\mathbf{D}(\rho)\left(\rho_{n}-\rho\right)$, and hence the second-order term scales as $\omega\left(\frac{1}{\sqrt{n}}\right)$ instead of the desired $\Theta\left(\frac{1}{\sqrt{n}}\right)$. To be more precise, because

$$
\Psi^{-1}(\mathbf{V}(\rho), \varepsilon) \subset\left[\begin{array}{c}
\sqrt{V_{1}(\rho)} \Phi^{-1}(\varepsilon) \\
\sqrt{V_{12}(\rho)} \Phi^{-1}(\varepsilon)
\end{array}\right]^{-},
$$

the bound in (110) implies that $\mathbf{R}_{n}$ must satisfy

$$
\mathbf{R}_{n} \in \mathbf{I}(\rho)+\mathbf{D}(\rho)\left(\rho_{n}-\rho\right)+\frac{1}{\sqrt{n}}\left[\begin{array}{c}
\sqrt{V_{1}(\rho)} \Phi^{-1}(\varepsilon) \\
\sqrt{V_{12}(\rho)} \Phi^{-1}(\varepsilon)
\end{array}\right]^{-}+o\left(\rho_{n}-\rho\right) \mathbf{1}
$$

Therefore, we have

$$
\mathbf{R}_{n} \leq \mathbf{I}(\rho)+\mathbf{D}(\rho)\left(\rho_{n}-\rho\right)+o\left(\rho_{n}-\rho\right) \mathbf{1}
$$

Since the first entry of $\mathbf{D}(\rho)$ is negative and the second entry is positive, (117) implies that at least one of the two lim inf values in (104) is equal to $-\infty$. That is, there are either no values of $L_{1}$ or no values of $L_{2}$ such that the desired second-order rate conditions are satisfied. We conclude that this case plays no role in the characterization of $\mathcal{L}$.

6) Completion of the Proof for Case (iii) $(\rho=1)$ : The case $\rho=1$ is handled in essentially the same way as $\rho \in(0,1)$, so we only state the differences. Since $\beta$ represents the difference between $\rho_{n}$ and $\rho$, and since $\rho_{n} \leq 1$, we should only consider the case that $\beta \leq 0$. Furthermore, for $\rho=1$ the set $\Psi^{-1}(\mathbf{V}(\rho), \varepsilon)$ can be written in a simpler form; see Lemma 6 Using this form, we readily obtain 45].

\section{B. Direct Part}

We obtain the local result from the global result using a similar (yet simpler) argument to the converse part in Section VII-A For fixed $\rho \in[0,1]$ and $\beta \in \mathbb{R}$, let

$$
\rho_{n}:=\rho+\frac{\beta}{\sqrt{n}},
$$


where we require $\beta \geq 0$ (resp. $\beta \leq 0$ ) when $\rho=0$ (resp. $\rho=1$ ). By Theorem 2 (global bound) and the definition of $R_{\text {in }}(n, \varepsilon ; \rho)$ in (34), rate pairs $\left(R_{1, n}, R_{2, n}\right)$ satisfying

$$
\mathbf{R}_{n} \in \mathbf{I}\left(\rho_{n}\right)+\frac{\Psi^{-1}\left(\mathbf{V}\left(\rho_{n}\right), \varepsilon\right)}{\sqrt{n}}+o\left(\frac{1}{\sqrt{n}}\right) \mathbf{1}
$$

are $(n, \varepsilon)$-achievable. Substituting (118) into (119) and performing Taylor expansions in an identical fashion to the converse part (cf. the argument from (108) to (110), we obtain

$$
\mathbf{R}_{n} \in \mathbf{I}(\rho)+\frac{\beta \mathbf{D}(\rho)}{\sqrt{n}}+\frac{\Psi^{-1}(\mathbf{V}(\rho), \varepsilon)}{\sqrt{n}}+o\left(\frac{1}{\sqrt{n}}\right) \mathbf{1} .
$$

We immediately obtain the desired result for case (ii) where $\rho \in[0,1)$. We also obtain the desired result for case (iii) where $\rho=1$ using the alternative form of $\Psi^{-1}(\mathbf{V}(1), \varepsilon)$ (see Lemma 6), similarly to the converse proof.

For case (i), we substitute $\rho=0$ into (40) and (41) to obtain $\mathbf{D}(\rho)=\left[\begin{array}{ll}0 & D_{12}(\rho)\end{array}\right]^{T}$ with $D_{12}(\rho)>0$. Since $\beta$ can be arbitrarily large, it follows from (120) that $L_{2}$ can take any real value. Furthermore, the set $\Psi^{-1}(\mathbf{V}(0), \varepsilon)$ contains vectors with a first entry arbitrarily close to $\sqrt{V_{1}(0)} \Phi^{-1}(\varepsilon)$ (provided that the other entry is sufficiently negative), and we thus obtain (43).

\section{APPENDIX A}

\section{MOMENTS OF THE INFORMATION DENSITY VECTOR}

Let $\rho \in[-1,1]$ be given, and recall the definition of the information density vector in (29), and the choices of $Q_{Y \mid X_{2}}$ and $Q_{Y}$ in (27)-(28). For a given pair of sequences $\left(\mathbf{x}_{1}, \mathbf{x}_{2}\right)$, form the random vector

$$
\mathbf{A}_{n}:=\frac{1}{\sqrt{n}} \sum_{i=1}^{n} \mathbf{j}\left(x_{1 i}, x_{2 i}, Y_{i}\right)
$$

where $Y_{i} \mid\left\{X_{1 i}=x_{1 i}, X_{2 i}=x_{2 i}\right\} \sim W\left(\cdot \mid x_{1 i}, x_{2 i}\right)$. Define the constants $\alpha:=S_{1}\left(1-\rho^{2}\right), \vartheta:=S_{1}+S_{2}+2 \rho \sqrt{S_{1} S_{2}}$ and $\kappa:=\rho \sqrt{S_{1} / S_{2}}$. Then, it can be verified that

$$
\begin{aligned}
& j_{1}\left(x_{1}, x_{2}, Y\right)=\frac{1}{2} \log (1+\alpha)-\frac{Z^{2}}{2}+\frac{\left(x_{1}-\kappa x_{2}+Z\right)^{2}}{2(1+\alpha)}=\frac{-\alpha Z^{2}+2\left(x_{1}-\kappa x_{2}\right) Z}{2(1+\alpha)}+f_{1}\left(x_{1}, x_{2}\right), \\
& j_{12}\left(x_{1}, x_{2}, Y\right)=\frac{1}{2} \log (1+\vartheta)-\frac{Z^{2}}{2}+\frac{\left(x_{1}+x_{2}+Z\right)^{2}}{2(1+\vartheta)}=\frac{-\vartheta Z^{2}+2\left(x_{1}+x_{2}\right) Z}{2(1+\vartheta)}+f_{12}\left(x_{1}, x_{2}\right),
\end{aligned}
$$

where $Z:=Y-x_{1}-x_{2} \sim \mathcal{N}(0,1)$ and $f_{1}\left(x_{1}, x_{2}\right)$ and $f_{12}\left(x_{1}, x_{2}\right)$ are deterministic functions that will not affect the covariance matrix. Taking the expectation, we obtain

$$
\begin{aligned}
\mathbb{E}\left[j_{1}\left(x_{1}, x_{2}, Y\right)\right] & =\frac{1}{2} \log (1+\alpha)-\frac{1}{2}+\frac{1+\left(x_{1}-\kappa x_{2}\right)^{2}}{2(1+\alpha)}=\frac{1}{2} \log (1+\alpha)+\frac{\left(x_{1}-\kappa x_{2}\right)^{2}-\alpha}{2(1+\alpha)}, \\
\mathbb{E}\left[j_{12}\left(x_{1}, x_{2}, Y\right)\right] & =\frac{1}{2} \log (1+\vartheta)-\frac{1}{2}+\frac{1+\left(x_{1}+x_{2}\right)^{2}}{2(1+\vartheta)}=\frac{1}{2} \log (1+\vartheta)+\frac{\left(x_{1}+x_{2}\right)^{2}-\vartheta}{2(1+\vartheta)} .
\end{aligned}
$$

Setting $x_{1} \leftarrow x_{1 i}, x_{2} \leftarrow x_{2 i}$ and $Y \leftarrow Y_{i}$ in (A.4) and A.5 and summing over all $i$, we conclude that the mean vector of $\mathbf{A}_{n}$ is

$$
\mathbb{E}\left[\mathbf{A}_{n}\right]=\sqrt{n}\left[\mathrm{C}(\alpha)+\frac{\left\|\mathbf{x}_{1}-\kappa \mathbf{x}_{2}\right\|_{2}^{2}-n \alpha}{2 n(1+\alpha)} \quad \mathrm{C}(\vartheta)+\frac{\left\|\mathbf{x}_{1}+\mathbf{x}_{2}\right\|_{2}^{2}-n \vartheta}{2 n(1+\vartheta)}\right]^{T} .
$$

From (A.2) and (A.3), we deduce that

$$
\begin{aligned}
\operatorname{Var}\left[j_{1}\left(x_{1}, x_{2}, Y\right)\right] & =\operatorname{Var}\left[\frac{-\alpha Z^{2}+2\left(x_{1}-\kappa x_{2}\right) Z}{2(1+\alpha)}\right]=\frac{\alpha^{2}+2\left(x_{1}-\kappa x_{2}\right)^{2}}{2(1+\alpha)^{2}}, \\
\operatorname{Var}\left[j_{12}\left(x_{1}, x_{2}, Y\right)\right] & =\operatorname{Var}\left[\frac{-\vartheta Z^{2}+2\left(x_{1}+x_{2}\right) Z}{2(1+\vartheta)}\right]=\frac{\vartheta^{2}+2\left(x_{1}+x_{2}\right)^{2}}{2(1+\vartheta)^{2}}
\end{aligned}
$$


where we have used $\operatorname{Var}\left[Z^{2}\right]=2$ and $\operatorname{Cov}\left[Z^{2}, Z\right]=\mathbb{E} Z^{3}-(\mathbb{E} Z)\left(\mathbb{E} Z^{2}\right)=0$. The covariance is

$$
\begin{aligned}
& \operatorname{Cov}\left[j_{1}\left(x_{1}, x_{2}, Y\right), j_{12}\left(x_{1}, x_{2}, Y\right)\right]=\operatorname{Cov}\left[\frac{-\alpha Z^{2}+2\left(x_{1}-\kappa x_{2}\right) Z}{2(1+\alpha)}, \frac{-\vartheta Z^{2}+2\left(x_{1}+x_{2}\right) Z}{2(1+\vartheta)}\right] \\
& =\frac{1}{4(1+\alpha)(1+\vartheta)}\left\{\mathbb{E}\left[\left(-\alpha Z^{2}+2\left(x_{1}-\kappa x_{2}\right) Z\right)\left(-\vartheta Z^{2}+2\left(x_{1}+x_{2}\right) Z\right)\right]\right. \\
& \left.-\mathbb{E}\left[-\alpha Z^{2}+2\left(x_{1}-\kappa x_{2}\right) Z\right] \mathbb{E}\left[-\vartheta Z^{2}+2\left(x_{1}+x_{2}\right) Z\right]\right\} \\
& =\frac{3 \alpha \vartheta+4\left(x_{1}-\kappa x_{2}\right)\left(x_{1}+x_{2}\right)-\alpha \vartheta}{4(1+\alpha)(1+\vartheta)}=\frac{\alpha \vartheta+2\left(x_{1}^{2}+(1-\kappa) x_{1} x_{2}-\kappa x_{2}^{2}\right)}{2(1+\alpha)(1+\vartheta)} .
\end{aligned}
$$

Setting $x_{1} \leftarrow x_{1 i}, x_{2} \leftarrow x_{2 i}$ and $Y \leftarrow Y_{i}$ in (A.7), (A.8) and (A.11) and summing over all $i$, we conclude that covariance matrix of $\mathbf{A}_{n}$ is

$$
\operatorname{Cov}\left[\mathbf{A}_{n}\right]=\left[\begin{array}{cc}
\frac{n \alpha^{2}+2\left\|\mathbf{x}_{1}-\kappa \mathbf{x}_{2}\right\|_{2}^{2}}{2 n(1+\alpha)^{2}} & \frac{n \alpha \vartheta+2\left(\left\|\mathbf{x}_{1}\right\|_{2}^{2}+(1-\kappa)\left\langle\mathbf{x}_{1}, \mathbf{x}_{2}\right\rangle-\kappa\left\|\mathbf{x}_{2}\right\|_{2}^{2}\right)}{2 n(1+\alpha)(1+\vartheta)} \\
\frac{n \alpha \vartheta+2\left(\left\|\mathbf{x}_{1}\right\|_{2}^{2}+(1-\kappa)\left\langle\mathbf{x}_{1}, \mathbf{x}_{2}\right\rangle-\kappa\left\|\mathbf{x}_{2}\right\|_{2}^{2}\right)}{2 n(1+\alpha)(1+\vartheta)} & \frac{n \vartheta^{2}+2\left\|\mathbf{x}_{1}+\mathbf{x}_{2}\right\|_{2}^{2}}{2 n(1+\vartheta)^{2}}
\end{array}\right] .
$$

In the remainder of the section, we analyze the third absolute moments associated with $\mathbf{A}_{n}$ appearing in the multivariate Berry-Esseen theorem [30], [31] (see Appendix B). The following lemma will be used to replace any given $\left(\mathbf{x}_{1}, \mathbf{x}_{2}\right)$ pair by an "equivalent" pair (in the sense that the statistics of $\mathbf{A}_{n}$ are unchanged) for which the corresponding third moments have the desired behavior. This is analogous to Polyanskiy et al. [8], where for the AWGN channel, one can use a spherical symmetry argument to replace any given sequence $\mathbf{x}$ such that $\|\mathbf{x}\|_{2}^{2}=n S$ with a fixed sequence $(\sqrt{S}, \cdots, \sqrt{S})$. In fact, this symmetry argument has been used by many other authors including Shannon [10].

Lemma 9. The joint distribution of $\mathbf{A}_{n}$ depends on $\left(\mathbf{x}_{1}, \mathbf{x}_{2}\right)$ only through the powers $\left\|\mathbf{x}_{1}\right\|_{2}^{2},\left\|\mathbf{x}_{2}\right\|_{2}^{2}$ and the inner product $\left\langle\mathbf{x}_{1}, \mathbf{x}_{2}\right\rangle$.

Proof: This follows by substituting (A.2)-A.3 into (A.1) and using the symmetry of the additive noise sequence $\mathbf{Z}=\left(Z_{1}, \ldots, Z_{n}\right)$. For example, from $(\mathrm{A} .2)$, the first entry of $\mathbf{A}_{n}$ can be written as

$$
\frac{1}{\sqrt{n}}\left(\frac{n}{2} \log (1+\alpha)-\frac{1}{2}\|\mathbf{Z}\|_{2}^{2}+\frac{1}{2(1+\alpha)}\left\|\mathbf{x}_{1}-\kappa \mathbf{x}_{2}+\mathbf{Z}\right\|_{2}^{2}\right)
$$

and the desired result follows by writing

$$
\left\|\mathbf{x}_{1}-\kappa \mathbf{x}_{2}+\mathbf{Z}\right\|^{2}=\left\|\mathbf{x}_{1}\right\|^{2}+\kappa^{2}\left\|\mathbf{x}_{2}\right\|^{2}+\|\mathbf{Z}\|^{2}-2 \kappa\left\langle\mathbf{x}_{1}, \mathbf{x}_{2}\right\rangle+2\left\langle\mathbf{x}_{1}-\kappa \mathbf{x}_{2}, \mathbf{Z}\right\rangle .
$$

Since $\mathbf{Z}$ is i.i.d. Gaussian (and in particular, circularly symmetric), the distribution of the final term depends on $\left(\mathbf{x}_{1}, \mathbf{x}_{2}\right)$ only through $\left\|\mathbf{x}_{1}-\kappa \mathbf{x}_{2}\right\|$, which in turn depends only on $\left\|\mathbf{x}_{1}\right\|_{2}^{2},\left\|\mathbf{x}_{2}\right\|_{2}^{2}$ and $\left\langle\mathbf{x}_{1}, \mathbf{x}_{2}\right\rangle$.

We now provide lemmas showing that, upon replacing a given pair $\left(\mathbf{x}_{1}, \mathbf{x}_{2}\right)$ with an equivalent pair using Lemma 9 if necessary, the corresponding third moments have the desired behavior. It will prove useful to work with the empirical correlation coefficient

$$
\rho_{\mathrm{emp}}\left(\mathbf{x}_{1}, \mathbf{x}_{2}\right):=\frac{\left\langle\mathbf{x}_{1}, \mathbf{x}_{2}\right\rangle}{\left\|\mathbf{x}_{1}\right\|_{2}\left\|\mathbf{x}_{2}\right\|_{2}}
$$

It is easily seen that Lemma 9 remains true when the inner product $\left\langle\mathbf{x}_{1}, \mathbf{x}_{2}\right\rangle$ is replaced by this normalized quantity.

Lemma 10. For any fixed $\tilde{\rho} \in[-1,1], S_{1}>0$ and $S_{2}>0$, there exists a sequence of pairs $\left(\mathbf{x}_{1}, \mathbf{x}_{2}\right)$ (indexed by increasing lengths $n$ ) such that $\left\|\mathbf{x}_{1}\right\|_{2}^{2}=n S_{1},\left\|\mathbf{x}_{2}\right\|_{2}^{2}=n S_{2}, \rho_{\mathrm{emp}}\left(\mathbf{x}_{1}, \mathbf{x}_{2}\right)=\tilde{\rho}$, and

$$
\tilde{T}_{n}:=\sum_{i=1}^{n} \mathbb{E}\left[\left\|\frac{1}{\sqrt{n}}\left(\mathbf{j}\left(x_{1 i}, x_{2 i}, Y_{i}\right)-\mathbb{E}\left[\mathbf{j}\left(x_{1 i}, x_{2 i}, Y_{i}\right)\right]\right)\right\|_{2}^{3}\right]=O\left(\frac{1}{\sqrt{n}}\right),
$$

where the $O\left(\frac{1}{\sqrt{n}}\right)$ term is uniform in $\tilde{\rho} \in[-1,1]$. 
Proof: Using the fact that $\|\mathbf{v}\|_{2} \leq\|\mathbf{v}\|_{1}$ and $(|a|+|b|)^{3} \leq 4|a|^{3}+4|b|^{3}$, we obtain

$$
\begin{aligned}
\tilde{T}_{n} \leq & \sum_{i=1}^{n} \mathbb{E}\left[\left\|\frac{1}{\sqrt{n}}\left(\mathbf{j}\left(x_{1 i}, x_{2 i}, Y_{i}\right)-\mathbb{E}\left[\mathbf{j}\left(x_{1 i}, x_{2 i}, Y_{i}\right)\right]\right)\right\|_{1}^{3}\right] \\
\leq 4 & \sum_{i=1}^{n} \mathbb{E}\left[\left|\frac{1}{\sqrt{n}}\left(j_{1}\left(x_{1 i}, x_{2 i}, Y_{i}\right)-\mathbb{E}\left[j_{1}\left(x_{1 i}, x_{2 i}, Y_{i}\right)\right]\right)\right|^{3}\right] \\
& +4 \sum_{i=1}^{n} \mathbb{E}\left[\left|\frac{1}{\sqrt{n}}\left(j_{12}\left(x_{1 i}, x_{2 i}, Y_{i}\right)-\mathbb{E}\left[j_{12}\left(x_{1 i}, x_{2 i}, Y_{i}\right)\right]\right)\right|^{3}\right] .
\end{aligned}
$$

We now specify $\left(\mathbf{x}_{1}, \mathbf{x}_{2}\right)$ whose powers and correlation match those given in the lemma statement. Assuming for the time being that $|\tilde{\rho}| \leq \frac{n-1}{n}$, we choose

$$
\begin{aligned}
& \mathbf{x}_{1}=\left(\sqrt{S_{1}}, \cdots, \sqrt{S_{1}}\right) \\
& \mathbf{x}_{2}=\left(\sqrt{S_{2}(1+\eta)}, \sqrt{S_{2}}, \cdots, \sqrt{S_{2}},-\sqrt{S_{2}(1-\eta)},-\sqrt{S_{2}}, \cdots,-\sqrt{S_{2}}\right),
\end{aligned}
$$

where $\eta \in(-1,1)$, and $\mathbf{x}_{2}$ contains $k \geq 1$ negative entries and $n-k \geq 1$ positive entries. It is easily seen that $\left\|\mathbf{x}_{1}\right\|_{2}^{2}=n S_{1}$ and $\left\|\mathbf{x}_{2}\right\|_{2}^{2}=n S_{2}$, as desired. Furthermore, we can choose $k$ and $\eta$ to obtain the desired correlation since

$$
\left\langle\mathbf{x}_{1}, \mathbf{x}_{2}\right\rangle=(n-2(k-1)+\sqrt{1+\eta}-\sqrt{1-\eta}) \sqrt{S_{1} S_{2}},
$$

and since the range of the function $f(\eta):=\sqrt{1+\eta}-\sqrt{1-\eta}$ for $\eta \in(-1,1)$ is given by $(-\sqrt{2}, \sqrt{2})$.

Using (A.2)-A.3), it can easily be verified that the third absolute moment of each entry of $\mathbf{j}\left(x_{1}, x_{2}, Y\right)$ (i.e. $\mathbb{E}\left|j_{1}\left(x_{1}, x_{2}, Y\right)-\mathbb{E}\left[j_{1}\left(x_{1}, x_{2}, Y\right)\right]\right|^{3}$ and $\left.\mathbb{E}\left|j_{12}\left(x_{1}, x_{2}, Y\right)-\mathbb{E}\left[j_{12}\left(x_{1}, x_{2}, Y\right)\right]\right|^{3}\right)$ is bounded above by some constant for any $\left(x_{1}, x_{2}\right)=\left(\sqrt{S_{1}}, \pm \sqrt{c S_{2}}\right)(c \in(0,2))$. We thus obtain (A.16) using (A.18). The proof is concluded by noting that a similar argument applies for the case $\tilde{\rho} \in\left(\frac{n-1}{n}, 1\right]$ by replacing A.20) by

$$
\mathbf{x}_{2}=\left(\sqrt{S_{2}(1+\eta)}, \sqrt{S_{2}(1-\eta)}, \sqrt{S_{2}}, \cdots, \sqrt{S_{2}}\right),
$$

and similarly (with negative entries) when $\tilde{\rho} \in\left[-1, \frac{n-1}{n}\right.$ ).

\section{APPENDIX B}

\section{A Multivariate BerRy-Esseen TheOrem}

In this section, we state a version of the multivariate Berry-Esseen theorem [30], [31] that is suited for our needs in this paper. The following is a restatement of Corollary 38 in [35].

Theorem 11. Let $\mathbf{U}_{1}, \ldots, \mathbf{U}_{n}$ be independent, zero-mean random vectors in $\mathbb{R}^{d}$. Let $\hat{\mathbf{Z}}_{n}:=\frac{1}{\sqrt{n}}\left(\mathbf{U}_{1}+\cdots+\mathbf{U}_{n}\right)$, Assume $\mathbf{V}:=\operatorname{Cov}\left(\hat{\mathbf{Z}}_{n}\right)$ is positive definite with minimum eigenvalue $\lambda_{\min }(\mathbf{V})>0$. Let $t:=\frac{1}{n} \sum_{i=1}^{n} \mathbb{E}\left[\left\|\mathbf{U}_{i}\right\|_{2}^{3}\right]$ and let $\mathbf{Z}$ be a zero-mean Gaussian random vector with covariance $\mathbf{V}$. Then, for all $n \in \mathbb{N}$,

$$
\sup _{\mathscr{C} \in \mathfrak{C}_{d}}\left|\operatorname{Pr}\left(\hat{\mathbf{Z}}_{n} \in \mathscr{C}\right)-\operatorname{Pr}(\mathbf{Z} \in \mathscr{C})\right| \leq \frac{k_{d} t}{\lambda_{\min }(\mathbf{V})^{3 / 2} \sqrt{n}},
$$

where $\mathfrak{C}_{d}$ is the family of all convex, Borel measurable subsets of $\mathbb{R}^{d}$, and $k_{d}$ is a function only of the dimension $d\left(\right.$ e.g., $\left.k_{2}=265\right)$.

\section{APPENDIX C}

PROOF OF LEMMA 5

Fix $\left(z_{1}, z_{2}\right) \in \Psi^{-1}\left(\mathbf{V}, \varepsilon+\lambda_{n}\right)$ and define $\mathbf{Z}=\left(Z_{1}, Z_{2}\right) \sim \mathcal{N}(\mathbf{0}, \mathbf{V})$. Since $\Psi^{-1}(\mathbf{V}, \varepsilon)$ is monotonic in the sense that $\Psi^{-1}(\mathbf{V}, \varepsilon) \subset \Psi^{-1}\left(\mathbf{V}, \varepsilon^{\prime}\right)$ for $\varepsilon \leq \varepsilon^{\prime}$, it suffices to verify that $\left(z_{1}, z_{2}\right)$ belongs to the set on the right-hand side of (71) for those $\left(z_{1}, z_{2}\right)$ on the boundary of $\Psi^{-1}\left(\mathbf{V}, \varepsilon+\lambda_{n}\right)$. That is (cf. (33)),

$$
\operatorname{Pr}\left(Z_{1} \leq-z_{1}, Z_{2} \leq-z_{2}\right)=1-\left(\varepsilon+\lambda_{n}\right)
$$


Define $\nu_{n}:=\inf \left\{\nu>0:\left(-z_{1}-\nu,-z_{2}-\nu\right) \in \Psi^{-1}(\mathbf{V}, \varepsilon)\right\}$. We need to show that $\nu_{n}=o(1)$ is bounded above by some linear function of $\lambda_{n}$. By using (C.1) and the definition of $\nu_{n}$, we see that

$$
\begin{aligned}
\lambda_{n} & =\operatorname{Pr}\left(Z_{1} \in\left[-z_{1}-\nu_{n},-z_{1}\right] \cup Z_{2} \in\left[-z_{2}-\nu_{n},-z_{2}\right]\right) \\
& \geq \max _{j=1,2}\left\{\Phi\left(\frac{-z_{j}}{\sqrt{V_{j j}}}\right)-\Phi\left(\frac{-z_{j}-\nu_{n}}{\sqrt{V_{j j}}}\right)\right\} .
\end{aligned}
$$

The assumption that $\mathbf{V}$ is a non-zero positive-semidefinite matrix ensures that at least one of $V_{j j}, j=1,2$ is non-zero. We have the lower bound

$$
\Phi\left(\frac{-z}{\sqrt{V}}\right)-\Phi\left(\frac{-z-\nu_{n}}{\sqrt{V}}\right) \geq \frac{\nu_{n}}{\sqrt{V}} \min \left\{\mathcal{N}(z ; 0, V), \mathcal{N}\left(z+\nu_{n} ; 0, V\right)\right\} .
$$

Hence, for all $n$ large enough, each of the terms in $\{\cdot\}$ in $(\mathbf{C . 3})$ is bounded below by $\nu_{n} f\left(z_{j}, V_{j j}\right)$ for $j=1,2$ where $f(z, V):=\frac{1}{2 \sqrt{V}} \mathcal{N}(z ; 0, V)$ satisfies $\lim _{z \rightarrow \pm \infty} f(z, V)=0$. Hence, $\nu_{n} \leq \lambda_{n} \min _{j=1,2}\left\{f\left(z_{j}, V_{j j}\right)^{-1}\right\}$. For every fixed $\varepsilon \in(0,1)$, every $\left(z_{1}, z_{2}\right) \in \Psi^{-1}\left(\mathbf{V}, \varepsilon+\lambda_{n}\right)$ satisfies $\min \left\{\left|z_{1}\right|,\left|z_{2}\right|\right\}<\infty$, and hence $\min _{j=1,2}\left\{f\left(z_{j}, V_{j j}\right)^{-1}\right\}$ is finite. This concludes the proof.

\section{APPENDIX D}

\section{PROOF OF LEMMA 6}

Recall that $\hat{\rho}_{n} \rightarrow 1$. We start by proving the inner bound on $\Psi^{-1}\left(\mathbf{V}\left(\hat{\rho}_{n}\right), \varepsilon\right)$. Let $\left(w_{1}, w_{2}\right)$ be an arbitrary element of the left-hand-side of (75), i.e. $w_{1} \leq-b_{n}$ and $w_{2} \leq \sqrt{V_{12}(1)} \Phi^{-1}\left(\varepsilon+a_{n}\right)-b_{n}$. Define the random variables $\left(Z_{1, n}, Z_{2, n}\right) \sim \mathcal{N}\left(\mathbf{0}, \mathbf{V}\left(\hat{\rho}_{n}\right)\right)$ and the sequence $b_{n}:=\left(1-\hat{\rho}_{n}\right)^{1 / 4}$. Consider

$$
\begin{aligned}
\operatorname{Pr}\left(Z_{1, n} \leq-w_{1}, Z_{2, n} \leq-w_{2}\right) & \geq \operatorname{Pr}\left(Z_{1, n} \leq b_{n}, Z_{2, n} \leq-\left(\sqrt{V_{12}(1)} \Phi^{-1}\left(\varepsilon+a_{n}\right)-b_{n}\right)\right) \\
& \geq \operatorname{Pr}\left(Z_{2, n} \leq-\left(\sqrt{V_{12}(1)} \Phi^{-1}\left(\varepsilon+a_{n}\right)-b_{n}\right)\right)-\operatorname{Pr}\left(Z_{1, n}>b_{n}\right) \\
& =\Phi\left(\frac{-\left(\sqrt{V_{12}(1)} \Phi^{-1}\left(\varepsilon+a_{n}\right)-b_{n}\right)}{\sqrt{V_{12}\left(\hat{\rho}_{n}\right)}}\right)-\Phi\left(\frac{-b_{n}}{\sqrt{V_{1}\left(\hat{\rho}_{n}\right)}}\right) .
\end{aligned}
$$

From the choice of $b_{n}$ and the fact that $\sqrt{V_{1}\left(\hat{\rho}_{n}\right)}=\Theta\left(\sqrt{1-\hat{\rho}_{n}}\right)$ (since $V_{1}(\rho)=\Theta(1-\rho)$ as $\rho \rightarrow 1$ by continuous differentiability), the argument of the second term scales as $-\left(1-\hat{\rho}_{n}\right)^{-1 / 4}$, which tends to $-\infty$. Hence, the second term vanishes. We may thus choose a vanishing sequence $a_{n}$ so that the expression in (D.3) equals $1-\varepsilon$. Such a choice satisfies $a_{n}=\Theta\left(b_{n}\right)=\Theta\left(\left(1-\hat{\rho}_{n}\right)^{1 / 4}\right)$, in accordance with the lemma statement. From the definition in (33), we have proved that $\left(w_{1}, w_{2}\right) \in \Psi^{-1}\left(\mathbf{V}\left(\hat{\rho}_{n}\right), \varepsilon\right)$ for this choice of $\left(a_{n}, b_{n}\right)$.

For the outer bound on $\Psi^{-1}\left(\mathbf{V}\left(\hat{\rho}_{n}\right), \varepsilon\right)$, let $\left(u_{1}, u_{2}\right)$ be an arbitrary element of $\Psi^{-1}\left(\mathbf{V}\left(\hat{\rho}_{n}\right), \varepsilon\right)$. By definition,

$$
\operatorname{Pr}\left(Z_{1, n} \leq-u_{1}, Z_{2, n} \leq-u_{2}\right) \geq 1-\varepsilon,
$$

where $\left(Z_{1, n}, Z_{2, n}\right) \sim \mathcal{N}\left(\mathbf{0}, \mathbf{V}\left(\hat{\rho}_{n}\right)\right)$ as above. Thus,

$$
1-\varepsilon \leq \operatorname{Pr}\left(Z_{2, n} \leq-u_{2}\right)=\Phi\left(\frac{-u_{2}}{\sqrt{V_{12}\left(\hat{\rho}_{n}\right)}}\right) .
$$

This leads to

$$
u_{2} \leq \sqrt{V_{12}\left(\hat{\rho}_{n}\right)} \Phi^{-1}(\varepsilon)=\sqrt{V_{12}(1)} \Phi^{-1}(\varepsilon)+c_{n}^{\prime}
$$

for some $c_{n}^{\prime}=\Theta\left(1-\hat{\rho}_{n}\right)$, since $\rho \mapsto \sqrt{V_{12}(\rho)}$ is continuously differentiable and its derivative does not vanish at $\rho=1$. Similarly, we have

$$
u_{1} \leq \sqrt{V_{1}\left(\hat{\rho}_{n}\right)} \Phi^{-1}(\varepsilon)=c_{n}^{\prime \prime}
$$

for some $c_{n}^{\prime \prime}=\Theta\left(\sqrt{1-\hat{\rho}_{n}}\right)$, since $V_{1}(1)=0$ and $\sqrt{V_{1}\left(\hat{\rho}_{n}\right)}=\Theta\left(\sqrt{1-\hat{\rho}_{n}}\right)$. Letting $c_{n}:=\max \left\{\left|c_{n}^{\prime}\right|,\left|c_{n}^{\prime \prime}\right|\right\}=$ $\Theta\left(\sqrt{1-\hat{\rho}_{n}}\right)$, we deduce that $\left(u_{1}, u_{2}\right)$ belongs to the rightmost set in (75). This completes the proof. 


\section{APPENDIX E}

\section{PROOF OF LEMMA 8}

Throughout the proof, we use the fact that for jointly Gaussian $\left(X_{1}, X_{2}\right)$ with powers $\left(S_{1}, S_{2}\right)$ and correlation $\rho$ (i.e. the covariance matrix given in (22)), we have

$$
X_{1} \mid\left\{X_{2}=x_{2}\right\} \sim \mathcal{N}\left(\rho \sqrt{\frac{S_{1}}{S_{2}}} x_{2}, S_{1}\left(1-\rho^{2}\right)\right) .
$$

Several aspects of the proof are similar to Polyanskiy et al. [8, Lem. 61] for the single-user setting, so we focus primarily on the parts that are different.

\section{A. Upper bounding $\Lambda_{1}$}

A straightforward symmetry argument reveals that

$$
\frac{\mathrm{d} P_{\mathbf{X}_{1} \mid \mathbf{X}_{2}} W^{n}\left(\cdot \mid \mathbf{x}_{2}\right)}{\mathrm{d} Q_{\mathbf{Y} \mid \mathbf{X}_{2}}\left(\cdot \mid \mathbf{x}_{2}\right)}(\mathbf{y})
$$

is the same for all $\mathbf{x}_{2}$ having a fixed magnitude. Since $\left\|\mathbf{X}_{2}\right\|_{2}^{2}=n S_{2}$ almost surely by construction, we focus on the convenient sequence $\mathbf{x}_{2}=\left(\sqrt{n S_{2}}, 0, \ldots, 0\right)$. The constraint $\left\langle\mathbf{x}_{1}, \mathbf{x}_{2}\right\rangle=n \rho \sqrt{S_{1} S_{2}}$ in (84) implies that the first entry of $\mathbf{x}_{1}$ equals $\rho \sqrt{n S_{1}}$ with probability one. Moreover, since $\left\|\mathbf{X}_{1}\right\|_{2}^{2}=n S_{1}$ almost surely, the remaining $(n-1)$ symbols must have a total power of $n S_{1}\left(1-\rho^{2}\right)$. Since (84) is the uniform distribution on the set satisfying the given conditions, we conclude that the final $(n-1)$ entries of $\mathbf{X}_{1}$ are uniform on the sphere of radius $\sqrt{n S_{1}\left(1-\rho^{2}\right)}$ centered at zero.

We wish to bound the Radon-Nikodym (RN) derivative of $\mathbf{Y}:=\mathbf{X}_{1}+\mathbf{x}_{2}+\mathbf{Z}$ with respect to $\mathbf{Y}^{\prime}:=\mathbf{X}_{1}^{\prime}+\mathbf{x}_{2}+\mathbf{Z}$, where $\mathbf{X}_{1}$ has the conditional distribution in (84), and $\mathbf{X}_{1}^{\prime}$ is i.i.d. on $P_{X_{1} \mid X_{2}} W$ given $\mathbf{x}_{2}$ (recall the choice of $Q_{\mathbf{Y} \mid \mathbf{X}_{2}}$ in the lemma statement). For notational convenience, we work with the vectors $\tilde{\mathbf{Y}}:=\mathbf{Y}-\mathbf{x}_{2}$ and $\tilde{\mathbf{Y}}^{\prime}:=\mathbf{Y}^{\prime}-\mathbf{x}_{2}$, we let $\tilde{\mathbf{y}}$ denote a generic sequence equaling $\mathbf{y}-\mathbf{x}_{2}$, and we write $\tilde{\mathbf{Y}}=\left(\tilde{Y}_{1}, \tilde{Y}_{2}^{n}\right)$ to split the first entry of $\tilde{\mathbf{Y}}$ from the other $(n-1)$ entries (and similarly for $\tilde{\mathbf{Y}}^{\prime}, \tilde{\mathbf{y}}$ and $\mathbf{Z}$ ). Since $\tilde{\mathbf{Y}}$ and $\tilde{\mathbf{Y}}^{\prime}$ are shifted versions of $\mathbf{Y}$ and $\mathbf{Y}^{\prime}$, it suffices to bound the RN derivative associated the former sequences. Observing that $\tilde{Y}_{1}$ is independent of $\tilde{Y}_{2}^{n}$ and similarly for $\tilde{\mathbf{Y}}^{\prime}$, we have

$$
\frac{\mathrm{d} P_{\tilde{\mathbf{Y}}}}{\mathrm{d} P_{\tilde{\mathbf{Y}}^{\prime}}}(\tilde{\mathbf{y}})=\frac{\mathrm{d} P_{\tilde{Y}_{1}}}{\mathrm{~d} P_{\tilde{Y}_{1}^{\prime}}}\left(\tilde{y}_{1}\right) \frac{\mathrm{d} P_{\tilde{Y}_{2}^{n}}}{\mathrm{~d} P_{\tilde{Y}_{2}^{\prime n}}}\left(\tilde{y}_{2}^{n}\right) .
$$

By (E.1) and the fact that the first entry of $\mathrm{x}_{2}$ equals $\sqrt{n S_{2}}$, the first RN derivative on the right-hand side equals the ratio of the densities $\mathcal{N}\left(\rho \sqrt{n S_{1}}, 1\right)$ and $\mathcal{N}\left(\rho \sqrt{n S_{1}}, 1+S_{1}\left(1-\rho^{2}\right)\right)$. Since the means coincide and the former has a smaller variance, this derivative is upper bounded by its value at the mean, which equals

$$
\frac{\frac{1}{\sqrt{2 \pi}}}{\frac{1}{\sqrt{2 \pi\left(1+S_{1}\left(1-\rho^{2}\right)\right)}}}=\sqrt{1+S_{1}\left(1-\rho^{2}\right)},
$$

and is thus uniformly bounded in $\rho \in[0,1]$.

We now handle the second term in (E.3), which is between the uniform distribution on the sphere of radius $\sqrt{n S}$ and the $(n-1)$-fold memoryless extension of $\mathcal{N}(0,1+S)$, where $S:=S_{1}\left(1-\rho^{2}\right)$. This is the same as the setting of [8, Lem. 61] other than two differences: (i) The block length is $n-1$ instead of $n$, so the radius $\sqrt{n S}$ is slightly larger than that which might be expected in analogy with [8], namely $\sqrt{(n-1) S}$. (ii) We must allow for all $S \in\left[0, S_{1}\right]$ (to accommodate for all $\rho \in[0,1]$ ), rather than considering only a fixed positive value. Fortunately, the proof of [8, Lem. 61] turns out to automatically handle both of these issues. Rather than repeating the proof here, we simply outline the differences.

We first note that, as in [8], we can restrict attention to sequences $\tilde{y}_{2}^{n}$ such that

$$
(n-1)(1+S-\delta) \leq\left\|\tilde{y}_{2}^{n}\right\|_{2}^{2} \leq(n-1)(1+S+\delta)
$$

for some $\delta \in(0,1)$, since the Chernoff bound implies that the probability of all remaining sequences vanishes exponentially fast, explaining the exponentially decaying term in (92). Note that the condition in (E.5) corresponds 
to the choice of $\mathcal{A}_{1}$ in the lemma statement; in the more general case where $\mathrm{x}_{2}$ may differ from $\left(\sqrt{n S_{2}}, 0, \ldots, 0\right)$, $\tilde{y}_{2}^{n}$ should be replaced by the projection of $\mathbf{y}$ onto the $(n-1)$-dimensional subspace orthogonal to $\mathbf{x}_{2}$.

Next, we observe that the second term in term in (E.3) depends on $\tilde{y}_{2}^{n}$ only through its squared magnitude $r:=\left\|\tilde{y}_{2}^{n}\right\|_{2}^{2}$. Thus, using [8, Eqs. (212)-(213)] to obtain explicit formulas for the densities of $\left\|\tilde{Y}_{2}^{n}\right\|_{2}^{2}$ and $\left\|\tilde{Y}_{2}^{\prime n}\right\|_{2}^{2}$, we obtain the following analog of [8, Eq. (426)]:

$$
\frac{\mathrm{d} P_{\tilde{Y}_{2}^{n}}}{\mathrm{~d} P_{\tilde{Y}_{2}^{\prime n}}}\left(\tilde{y}_{2}^{n}\right)=(1+S)^{\frac{n^{\prime}}{2}} \exp \left(-n^{\prime} \frac{S}{2}-r \frac{S}{2(S+1)}\right)\left(\left(n^{\prime}+1\right) S r\right)^{-\frac{1}{2}\left(\frac{n^{\prime}}{2}-1\right)} 2^{\frac{n^{\prime}}{2}} \Gamma\left(\frac{n^{\prime}}{2}\right) I_{n^{\prime} / 2-1}\left(\sqrt{\left(n^{\prime}+1\right) S r}\right),
$$

where $I_{k}(z)$ is the modified Bessel function of the first kind, $\Gamma(\cdot)$ is the Gamma function, and we have written $n^{\prime}:=n-1$ for the sake of ease of comparison with [8, Lem. 61]. The desired result is now obtained as in [8, Lem. 61] by upper bounding the Gamma function and Bessel function using [8, Eq. (428)] and [8, Eq. (430)] (the former of which should be combined with $\sinh ^{-1}(z)=\log \left(z+\sqrt{1+z^{2}}\right)$ ) and applying algebraic manipulations.

To gain some intuition as to why arbitrarily small values of $S$ are permitted (which is the main difference in our analysis compared to [8]), one may consider the case $S=0$, corresponding to $\rho=1$. This case is trivial, since it yields $\tilde{Y}_{2}^{n}=Z_{2}^{n}$ and $\tilde{Y}_{2}^{\prime n}=Z_{2}^{n}$ with probability one, thus yielding an RN derivative of one.

\section{B. Upper bounding $\Lambda_{12}$}

Observe that, by construction in (83)-(84), we have $\left\|\mathbf{X}_{1}+\mathbf{X}_{2}\right\|_{2}^{2}=n S_{1}+n 2 \rho \sqrt{S_{1} S_{2}}+n S_{2}$ with probability one. Thus, by symmetry, $\mathbf{X}_{1}+\mathbf{X}_{2}$ is uniform on the sphere of radius $\sqrt{n\left(S_{1}+S_{2}+2 \rho \sqrt{S_{1} S_{2}}\right)}$, and the RN derivative we seek is identical to that characterized in the proof of [8, Lem. 61]. Thus, the desired result follows by choosing $\mathcal{A}_{12}$ in the same way as [8, Eq. (416)]:

$$
\mathcal{A}_{12}=\left\{\mathbf{y}: n\left(1+S_{1}+S_{2}+2 \rho \sqrt{S_{1} S_{2}}-\delta\right) \leq\|\mathbf{y}\|_{2}^{2} \leq n\left(1+S_{1}+S_{2}+2 \rho \sqrt{S_{1} S_{2}}+\delta\right)\right\}
$$

for some $\delta \in(0,1)$.

\section{ACKNOWLEDGMENT}

We are grateful to Ebrahim MolavianJazi for pointing us to a minor error in an earlier version of the paper.

This first author has been funded in part by the European Research Council under ERC grant agreement 259663, by the European Union's 7th Framework Programme under grant agreement 303633, and by the Spanish Ministry of Economy and Competitiveness under grant TEC2012-38800-C03-03. The second author has been supported by A*STAR and NUS grants R-263-000-A98-750/133.

\section{REFERENCES}

[1] A. El Gamal and Y.-H. Kim, Network Information Theory. Cambridge, U.K.: Cambridge University Press, 2012.

[2] T. Cover, "Broadcast channels," IEEE Trans. on Inf. Th., vol. 18, no. 1, pp. 2-14, 1972.

[3] M. Hayashi, "Second-order asymptotics in fixed-length source coding and intrinsic randomness," IEEE Trans. on Inf. Th., vol. 54, pp. 4619-37, Oct 2008.

[4] M. Hayashi, "Information spectrum approach to second-order coding rate in channel coding," IEEE Trans. on Inf. Th., vol. 55, pp. 494766, Nov 2009.

[5] R. Nomura and T. S. Han, "Second-order Slepian-Wolf coding theorems for non-mixed and mixed sources," IEEE Trans. on Inf. Th., vol. 60, pp. 5553-5572, Sep 2014.

[6] R. Nomura and T. S. Han, "Second-order resolvability, intrinsic randomness, and fixed-length source coding for mixed sources: Information spectrum approach," IEEE Trans. on Inf. Th., vol. 59, pp. 1-16, Jan 2013.

[7] V. Strassen, “Asymptotische Abschätzungen in Shannons Informationstheorie," in Trans. Third Prague Conf. Inf. Theory, (Prague), pp. 689-723, 1962.

[8] Y. Polyanskiy, H. V. Poor, and S. Verdú, "Channel coding in the finite blocklength regime," IEEE Trans. on Inf. Th., vol. 56, pp. 23072359, May 2010.

[9] V. Y. F. Tan and M. Tomamichel, "The third-order term in the normal approximation for the AWGN channel," IEEE Trans. on Inf. Th., vol. 61, pp. 2430-2438, May 2015.

[10] C. E. Shannon, "Probability of error for optimal codes in a Gaussian channel," Bell Systems Technical Journal, vol. 38, pp. 611-656, 1959.

[11] V. Y. F. Tan and O. Kosut, "On the dispersions of three network information theory problems," IEEE Trans. on Inf. Th., vol. 60, no. 2, pp. 881-903, 2014.

[12] Y.-W. Huang and P. Moulin, "Finite blocklength coding for multiple access channels," in Int. Symp. Inf. Th., (Boston, MA), 2012. 
[13] E. MolavianJazi and J. N. Laneman, "Simpler achievable rate regions for multiaccess with finite blocklength," in Int. Symp. Inf. Th., (Boston, MA), 2012.

[14] E. MolavianJazi and J. N. Laneman, "A random coding approach to Gaussian multiple access channels with finite blocklength," in Allerton Conference, (Monticello, IL), 2012.

[15] E. MolavianJazi and J. N. Laneman, "A finite-blocklength perspective on Gaussian multi-access channels," arXiv:1309.2343 [CS.IT], Sep 2013.

[16] S. Verdú, "Non-asymptotic achievability bounds in multiuser information theory," in Allerton Conference, (Monticello, IL), 2012.

[17] P. Moulin, "A new metaconverse and outer region for finite-blocklength MACs," in Info. Th. and Applications (ITA) Workshop, (San Diego, CA), 2013.

[18] J. Scarlett, A. Martinez, and A. Guillén i Fàbregas, "Second-order rate region of constant-composition codes for the multiple-access channel," IEEE Trans. on Inf. Th., vol. 61, no. 1, pp. 157-172, 2015.

[19] E. Haim, Y. Kochman, and U. Erez, "A note on the dispersion of network problems," in Convention of Electrical and Electronics Engineers in Israel (IEEEI), 2012.

[20] V. Y. F. Tan, "Asymptotic estimates in information theory with non-vanishing error probabilities," Foundations and Trends in Communications and Information Theory, vol. 11, no. 1-2, pp. 1-183, 2014.

[21] S. I. Bross, A. Lapidoth, and M. Wigger, "Dirty-paper coding for the Gaussian multiaccess channel with conferencing," IEEE Trans. on Inf. Th., vol. 58, no. 9, pp. 5640-5668, 2012.

[22] R. Ahlswede, "An elementary proof of the strong converse theorem for the multiple access channel," J. of Combinatorics, Information \& System Sciences, pp. 216-230, 1982

[23] J. Scarlett and V. Y. F. Tan, "Second-order asymptotics for the discrete memoryless MAC with degraded message sets," in Intl. Symp. Info. Th., (Hong Kong), June 2015.

[24] I. Csiszár and J. Körner, Information Theory: Coding Theorems for Discrete Memoryless Systems. Cambridge University Press, 2011.

[25] Y. Polyanskiy, Channel coding: Non-asymptotic fundamental limits. PhD thesis, Princeton University, 2010.

[26] G. Dueck, "Maximal error capacity regions are smaller than average error capacity regions for multi-user channels," Probl. Control Inf. Theory, vol. 7, pp. 11-19, 1978.

[27] T. S. Han, "An information-spectrum approach to capacity theorems for the general multiple-access channel," IEEE Trans. on Inf. Th., vol. 44, pp. 2773-2795, Jul 1998.

[28] S. Boucheron and M. R. Salamatian, "About priority encoding transmission," IEEE Trans. on Inf. Th., vol. 46, no. 2, pp. 699-705, 2000.

[29] M. Hayashi and H. Nagaoka, "General formulas for capacity of classical-quantum channels," IEEE Trans. on Inf. Th., vol. 49, pp. 17531768, Jul 2003

[30] F. Götze, "On the rate of convergence in the multivariate CLT," The Annals of Probability, vol. 19, no. 2, pp. 721-739, 1991.

[31] R. Bhattacharya and S. Holmes, "An exposition of Götze's estimation of the rate of convergence in the multivariate central limit theorem," tech. rep., Stanford University, 2010. arxiv:1003.4254 [math.ST] .

[32] J. N. Laneman, "On the distribution of mutual information," in Information Theory and Applications Workshop, 2006.

[33] W. Feller, An Introduction to Probability Theory and Its Applications. John Wiley and Sons, 2nd ed., 1971.

[34] W. Rudin, Principles of Mathematical Analysis. McGraw-Hill, 1976.

[35] S. Watanabe, S. Kuzuoka, and V. Y. F. Tan, "Non-asymptotic and second-order achievability bounds for coding with side-information," IEEE Trans. on Inf. Th., vol. 61, pp. 1574-1605, Apr 2015.

Jonathan Scarlett (S'14-M'15) was born in Melbourne, Australia, in 1988. In 2010, he received the B.Eng. degree in electrical engineering and the B.Sci. degree in computer science from the University of Melbourne, Australia. In 2011, he was a research assistant at the Department of Electrical \& Electronic Engineering, University of Melbourne. From October 2011 to August 2014, he was a Ph.D. student in the Signal Processing and Communications Group at the University of Cambridge, United Kingdom. He is now a post-doctoral researcher with the Laboratory for Information and Inference Systems at the École Polytechnique Fédérale de Lausanne, Switzerland. His research interests are in the areas of information theory, signal processing, and high-dimensional statistics. He received the Poynton Cambridge Australia International Scholarship, and the 'EPFL Fellows' postdoctoral fellowship co-funded by Marie Curie.

Vincent Y. F. Tan (S'07-M'11-SM'15) is an Assistant Professor in the Department of Electrical and Computer Engineering (ECE) and the Department of Mathematics at the National University of Singapore (NUS). He received the B.A. and M.Eng. degrees in Electrical and Information Sciences from Cambridge University in 2005 and the Ph.D. degree in Electrical Engineering and Computer Science (EECS) from the Massachusetts Institute of Technology in 2011. He was a postdoctoral researcher in the Department of ECE at the University of Wisconsin-Madison and a research scientist at the Institute for Infocomm $\left(I^{2} R\right)$ Research, A*STAR, Singapore. His research interests include information theory, machine learning and signal processing.

Dr. Tan received the MIT EECS Jin-Au Kong outstanding doctoral thesis prize in 2011 and the NUS Young Investigator Award in 2014. $\mathrm{He}$ is currently an Editor of the IEEE Transactions on Communications. 